"This document is the Accepted Manuscript version of a Published Work that appeared in final form in The Journal of Physical Chemistry A, copyright $(\mathcal{C}$ American Chemical Society after peer review and technical editing by the publisher. To access the final edited and published work see https://pubs-acs-org.uml.idm.oclc.org/doi/10.1021/acs.jpca.5b07379”.

\title{
Investigation of Structural Trends in Mono-, Di- and Pentafluorobenzonitriles Using Fourier Transform Microwave Spectroscopy
}

Mahdi Kamaee, ${ }^{1}$ Ming Sun, ${ }^{2}$ Horace Luong ${ }^{1}$ and Jennifer van Wijngaarden ${ }^{1 *}$

${ }^{1}$ Department of Chemistry, University of Manitoba, Winnipeg Manitoba, R3T 2N2 Canada

${ }^{2}$ School of Electronic and Optical Engineering, Nanjing University of Science and Technology, Xiao Lingwei Road 200, Nanjing, Jiangsu, 210094 China

${ }^{*}$ Corresponding author

Email: vanwijng@cc.umanitoba.ca

Phone: (204)474-8379

Fax: (204)474-7608 


\begin{abstract}
The ground state rotational spectra of a series of fluorinated benzonitriles (BN), namely: 2fluorobenzonitrile (2FBN), 3-fluorobenzonitrile (3FBN), 2,3-difluorobenzonitrile (23DFBN), 2,4-difluorobenzonitrile (24DFBN) and pentafluorobenzonitrile (PFBN), have been investigated between 4 and $24 \mathrm{GHz}$ using Fourier transform microwave (FTMW) spectroscopy. The assigned transitions include those due to the parent as well as the ${ }^{13} \mathrm{C}$ and ${ }^{15} \mathrm{~N}$ singly-substituted isotopologues which were observed in natural abundance. The spectroscopic analysis allowed the derivation of substitution $\left(\mathrm{r}_{\mathrm{s}}\right)$ and effective ground state structures $\left(\mathrm{r}_{0}\right)$ to investigate the effect of mono-, di- and pentafluoro substitution on the geometry of the BN backbone and are compared here with $a b$ initio values of the equilibrium parameters $\left(\mathrm{r}_{\mathrm{e}}\right)$ obtained from MP2/6-311++G(2d,2p) calculations. Analysis of the ${ }^{14} \mathrm{~N}$ hyperfine structure provides additional information about the electronic structure surrounding the nitrogen atom. The observed geometry changes relative to the reference $\mathrm{BN}$ compound are interpreted using natural bond orbital (NBO) analysis to describe differences in the hybridization at various sites and contributions from plausible resonance structures.
\end{abstract}




\section{A. Introduction}

Fluorine substitution has been shown to have significant effects on a compound's physical and chemical properties as it is a much heavier, more electronegative atom than hydrogen. The C-F bond is the strongest single bond ${ }^{1}$ in organic chemistry and the unique chemistry of fluorinated compounds has been used to tune the properties of materials, ${ }^{2}$ pharmaceuticals ${ }^{3}$ and agrochemicals. ${ }^{4}$ In addition to serving as an electron withdrawing substituent, one lone pair of electrons on the fluorine atom is also oriented such that it can donate electron density into adjacent $\pi$-orbitals through hyperconjugation. A number of fluorinated aromatic compounds have been investigated via high resolution microwave spectroscopy with the aim of providing a better understanding of the influence of fluorination on the molecular geometry. These include, but are not limited to, the effect of fluorine substitution on the aromatic backbone in fluorobenzene ${ }^{5,6,7}$ and fluoropyridine. ${ }^{8,9}$ In the monofluorinated species, the largest geometric changes occur near the site of fluorination with an increase in the ring angle at that site by $3-4^{\circ}$ and a shortening of the adjacent $\mathrm{C}-\mathrm{C}$ bonds by $0.006-0.010 \AA$. This was attributed to inductive effects due to the electronegative fluorine. The shortening of certain bond lengths in the aromatic backbone is also consistent with an increase in $\pi$-electron density arising from hyperconjugation involving the fluorine substituent and were supported by calculated electrostatic potential surfaces in the case of the fluoropyridines. ${ }^{8}$ 
In this article, we describe our recent systematic investigation of the effect of fluorine substituents at various sites on a benzonitrile (cyanobenzene) ring. The presence of a cyano substituent in tandem with fluorine introduces the possibility of additional mesomeric and inductive effects on the aromatic backbone which may be probed using the ${ }^{14} \mathrm{~N}$ nuclear quadrupole interaction of the cyano group. Benzonitrile $(\mathrm{BN})$ itself has an appreciable dipole moment (4.5152(58) D) ${ }^{10}$ and its size makes it highly amenable to high level ab initio calculations of its properties. ${ }^{11,12}$ Experimentally, BN has been the subject of numerous microwave spectroscopic investigations over the past sixty years. ${ }^{13,14,15}$ Spectra of the ${ }^{13} \mathrm{C}$ isotopic species ${ }^{16,17}$ allowed estimation of its substitution $\left(r_{s}\right)$ structure. Dreizler and coworkers ${ }^{18,19}$ were the first to report the ${ }^{14} \mathrm{~N}$ nuclear quadrupole coupling constants using Fourier transform microwave (FTMW) spectroscopy and provided an updated $r_{s}$ structure. ${ }^{20}$ Recent high resolution microwave studies have settled discrepancies in the magnitude of the dipole moment ${ }^{10}$ and have demonstrated that benzonitrile is a prototypical polyatomic for controlling population of rotational states (including complete population inversion) $)^{21}$ and an attractive candidate for deceleration and trapping experiments..$^{22,23}$

Among the fluorinated variants of $\mathrm{BN}$, the parent isotopologue of pentafluorobenzonitrile (PFBN) was the first to be investigated via Stark modulated microwave spectroscopy ${ }^{24}$ and its spectrum was later re-investigated with higher resolution using FTMW spectroscopy for analysis of the ${ }^{14} \mathrm{~N}$ hyperfine structure. ${ }^{25}$ The monofluorinated species having substituents at the ortho, ${ }^{26,27,28}$ meta $^{28,29}$ and para $^{28,30,31}$ positions relative to $\mathrm{CN}$ have been investigated in microwave waveguides and for 2-fluorobenzonitirile, the rotational spectrum is known into the millimeterwave region $(99 \mathrm{GHz}){ }^{32}$ Although minor isotopologues were not observed, the resolution of ${ }^{14} \mathrm{~N}$ hyperfine structure provided useful information about the electron density 
around the cyano group as a function of the fluorine position. It was reported that when the fluorine substitution is ortho or para to the cyano group, there is more electron density in the $\pi$ orbitals perpendicular to the molecular plane $\left(\pi_{\mathrm{y}}\right)$ on nitrogen in comparison to the case in which the substituents are in a meta arrangement. This was attributed to additional resonance structures in the ortho and para species that have cumulenic $\mathrm{C}=\mathrm{C}=\mathrm{N}$ linkages. ${ }^{28}$ For the difluorinated moieties, the literature is sparse. The rotational spectra of both 2,3-difluoro- ${ }^{33,34}$ and 2,6difluorobenzonitrile ${ }^{35}$ were reported although only the former was studied with sufficient resolution to estimate ${ }^{14} \mathrm{~N}$ quadrupole coupling constants. ${ }^{36}$ To the best of our knowledge, there are no previous microwave spectroscopic studies of other di-, tri-, and tetra- fluorinated benzonitriles and no reports of the ${ }^{13} \mathrm{C}$ and ${ }^{15} \mathrm{~N}$ analogues of any of the aforementioned fluorinated species for the purpose of structural investigation. The aim of the present systematic study is to elucidate the effect of fluorination on the ring geometry as a function of the substitution site relative to other electron withdrawing groups ( $\mathrm{F}$ or $\mathrm{CN})$.

In this paper, we report high resolution pulsed-jet FTMW spectra from 4-24 GHz of the ${ }^{13} \mathrm{C}$ and ${ }^{15} \mathrm{~N}$ singly-substituted variants of pentafluorobenzonitrile (PFBN), 2-fluorobenzonitrile (2FBN), 3-fluorobenzonitrile (3FBN), 2,3-difluorobenzonitrile (23DFBN) and 2,4difluorobenzonitrile $(24 \mathrm{DFBN})$ for the first time as well as extend the list of observed transitions for isotopologues of benzonitrile (BN). In order to have a complete dataset from which to derive accurate structural parameters, we also re-measured previously reported transitions of the parent species of each compound and extended the observation range to lower frequencies $(4 \mathrm{GHz})$ in many cases. The spectrum of the parent $24 \mathrm{DFBN}$ is reported here for the first time. The rotational constants of all species were used to derive geometric parameters using both Kraitchmann analyses $\left(\mathrm{r}_{\mathrm{s}}\right)$ and least squares fits of the moments of inertia $\left(\mathrm{r}_{\mathrm{o}}\right)$. The ${ }^{14} \mathrm{~N}$ hyperfine 
transitions provide additional information from which to interpret electronic structure changes in the compounds under study. The results of these analyses are in favourable agreement with $a b$ initio calculations (MP2, 6-311++G(2d, 2p)) in this work $\left(\mathrm{r}_{\mathrm{e}}\right)$ and consistent with interpretation based on natural bond orbital (NBO) analysis.

\section{B. Experimental}

The benzonitriles chosen for this study are commercially available with sufficient vapour pressure for spectroscopic study. Samples of BN (99\%), PFBN (99\%), 2FBN (98\%), 3FBN (98\%), 23DFBN (98\%) and 24DFBN (97\%) were purchased from Sigma-Aldrich and used without further purification. As the first five samples are high boiling liquids (boiling points greater than $162^{\circ} \mathrm{C}$ ), a few millimeters of each substance were placed in a glass vessel and approximately 1 bar of carrier gas ( $\mathrm{Ne}$ or Ar) was continuously bubbled through the liquid before the mixture was expanded into the vacuum chamber via a pulsed nozzle. The glass vessel was seated in a heating bath that was maintained between $40-60^{\circ} \mathrm{C}$ to increase the vapour pressure of the compound of interest. As $24 \mathrm{DFBN}$ is a solid $\left(\mathrm{mp} 47-49^{\circ} \mathrm{C}\right)$, a similar procedure was followed except that the compound was melted in the vessel before the gas was bubbled through. The spectra of all minor isotopologues were observed in natural abundance $\left({ }^{13} \mathrm{C}-1.07 \%,{ }^{15} \mathrm{~N}-\right.$ $0.36 \%$ ). Rotational transitions of each compound were primarily collected using a Balle-Flygare FTMW spectrometer which has been previously described. ${ }^{37}$ The high resolution (FWHM $\sim 7$ $\mathrm{kHz}$ ) was necessary for elucidating the hyperfine splittings due to the ${ }^{14} \mathrm{~N}$ quadrupole moment. For compounds for which there were not previous microwave studies as a starting point, including 24DFBN and the minor isotopologues of species such as PFBN, a chirp FTMW spectrometer ${ }^{38}$ was first used to record a series of $2 \mathrm{GHz}$ surveys with lower resolution $(\sim 100$ 
$\mathrm{kHz}$ ). These broadband spectra served as a guide for subsequent high resolution measurements using the cavity-based spectrometer.

\section{Spectral assignment and analysis}

i) Benzonitrile (BN) and pentafluorbenzonitrile (PFBN)

The dipole moment of BN is well-established experimentally $(4.5152(58) \mathrm{D})^{10}$ and the $a$ type rotational spectrum of the parent compound has been extensively reported as described above. Owing to its $\mathrm{C}_{2 \mathrm{v}}$ symmetry as shown in Figure 1, there are five unique carbon atoms in $\mathrm{BN}$ for heavy atom substitution as well as a single nitrogen atom. Although the ${ }^{13} \mathrm{C}$ isotopic species were previously investigated, earlier studies either did not have sufficient resolution for ${ }^{14} \mathrm{~N}$ hyperfine analysis ${ }^{16,17}$ or were limited in range $(6-11 \mathrm{GHz})$ and did not provide lists of transitions ${ }^{20}$ As the aim of our study is to identify geometry changes that accompany fluorination at various sites of the BN ring, we thought it important to start with a set of comparable spectroscopic parameters for $\mathrm{BN}$ itself and thus decided to re-investigate the microwave spectrum of $\mathrm{BN}$ and its ${ }^{13} \mathrm{C}$ and ${ }^{15} \mathrm{~N}$ analogues in the region of 4-24 GHz. The complete list of assigned transitions is provided as Supporting Information. The transitions were fit using Pickett's SPFIT program (Watson's A-reduced Hamiltonian, Ir representation) ${ }^{39}$ and the resulting spectroscopic constants including ${ }^{14} \mathrm{~N}$ quadrupole coupling constants are reported in Table 1. For the minor isotopologues, the $\delta_{\mathrm{K}}$ centrifugal distortion constant was held fixed to the value obtained for the parent compound because fewer transitions were recorded that were sensitive to this parameter.

PFBN has $\mathrm{C}_{2 \mathrm{~V}}$ symmetry and an estimated dipole moment of $2.7864 \mathrm{D}$ (Gaussian $09,{ }^{40}$ MP2-6-311++G(2d,2p), this work) along the a-principal inertial axis as shown in Figure 1. Using 
the rotational constants reported by M. Krüger and H. Dreizler ${ }^{25}$ for the parent species and the $a b$ initio geometry as a guide, we first investigated PFBN using survey scans between 8 and $14 \mathrm{GHz}$ using the cp-FTMW instrument to identify transitions from the five unique ${ }^{13} \mathrm{C}$ and the ${ }^{15} \mathrm{~N}$ isotopologues which were not previously reported. A portion of a broadband spectrum is shown in Figure 2. Once a preliminary assignment was complete, individual rotational transitions and their ${ }^{14} \mathrm{~N}$ hyperfine structure were sought using the Balle-Flygare instrument from 4-24 GHz and a sample spectrum of the $10_{0} 10^{-} 9_{0}$ transition $\left(\mathrm{J}_{\mathrm{KaKc}}\right)$ is provided in Figure 3. Due to the greater mass of the pentafluorinated ring, a larger number of transitions fall within the range of the spectrometers compared with BN. The full list of observed transitions is provided as Supporting Information. The transitions were fit as described for $\mathrm{BN}$ and the resulting spectroscopic constants are reported in Table 2 along with ab initio values for comparison. For the minor isotopologues, three centrifugal distortion constants $\left(\Delta_{\mathrm{K}}, \delta_{\mathrm{J}}, \delta_{\mathrm{K}}\right)$ were held fixed to the values obtained for the parent compound. The ${ }^{14} \mathrm{~N}$ nuclear quadrupole coupling constants were estimated using both MP2 and DFT/B3LYP calculations (basis set $6.311++\mathrm{G}(2 \mathrm{~d}, 2 \mathrm{p})$ ) with the latter showing better agreement with the experimental values. The inertial defects $\left(\Delta_{0}\right)$ of BN (0.0801 amu $\left.\AA^{2}\right)$ and PFBN (-0.00111 amu $\left.\AA^{2}\right)$ are small as one would expect for planar molecules.

\section{ii) 2-fluorobenzonitrile (2FBN) and 3-fluorobenzonitrile (3FBN)}

The $2 \mathrm{FBN}$ and 3FBN molecules are shown in their principal inertial axis systems in Figure 1. The rotational spectrum of $2 \mathrm{FBN}$ is composed of $a$ - and $b$-type transitions as there are appreciable dipole components along both axes of the molecular plane $\left(\mu_{\mathrm{a}}=5.97 \mathrm{D}, \mu_{\mathrm{b}}=-0.81 \mathrm{D}\right.$ from MP2 (6-311++G(2d,2p)) calculations, this work). The same holds true for $3 \mathrm{FBN}$ as the 
estimated values are $\mu_{\mathrm{a}}=3.45 \mathrm{D}, \mu_{\mathrm{b}}=-2.64 \mathrm{D}$ (this work). Due to reduced symmetry, there are seven unique carbon atoms in each of these compounds leading to a total of eight observable minor isotopologues $\left({ }^{13} \mathrm{C},{ }^{15} \mathrm{~N}\right)$ in addition to the parent species previously studied. ${ }^{26,27,28,29}$ The observed transitions are compiled in the Supporting Information and the resulting spectroscopic constants are given in Tables 3 and 4 for 2FBN and 3FBN, respectively. As fewer transitions were observed for the minor species, some centrifugal distortion parameters were held fixed to the values obtained from fitting the parent spectra as indicated in the tables. Certain transitions of 3FBN were found to be sensitive to the inclusion of the off-diagonal component of the quadrupole coupling tensor $\left(\chi_{\mathrm{ab}}\right)$; it was well-determined in the fit $(1.8608(27) \mathrm{MHz})$ and comparable to the ab initio value $1.875 \mathrm{MHz}$ (DFT). For 2FBN, however, the observed transitions were not sensitive to this parameter; perhaps because it is smaller in magnitude ($0.837 \mathrm{MHz})(\mathrm{DFT})$. Fixing $\chi_{\mathrm{ab}}$ to the calculated value did not influence the overall fit and it was thus not included in the final analysis. The inertial defects are $0.117 \mathrm{amu} \AA^{2}$ and $0.115 \mathrm{amu} \AA^{2}$ for $2 \mathrm{FBN}$ and $3 \mathrm{FBN}$, respectively.

\section{iii) 2,3-difluorobenzonitrile (23DFBN) and 2,4-difluorobenzonitrile (24DFBN)}

Both 23DFBN and 24DFBN are shown in their principal axis systems in Figure 1 and their rotational spectra are comprised of $a$ - and $b$-type transitions. The estimated dipole components along the $\mathrm{a}-$ and $\mathrm{b}$-axes are $-3.39 \mathrm{D}$ and $-4.03 \mathrm{D}$, respectively for 23DFBN and -4.13 D and -1.08 D for 24DFBN, respectively from MP2 $(6-311++G(2 d, 2 p))$ calculations (this work). Owing to the smaller $\mu_{\mathrm{b}}$ dipole component for $24 \mathrm{DFBN}$, fewer $b$-type rotational transitions were measured for the parent and none were observed for the eight minor isotopologues. The full set of assigned lines for both species are compiled in the Supporting Information and the resulting 
spectroscopic constants are given in Tables 5 and 6 for 23DFBN and 24DFBN, respectively. As fewer transitions were observed for the minor species, some centrifugal distortion parameters were held fixed to the values obtained from fitting the parent spectra as indicated in the tables. The spectra were not sensitive to the inclusion of $\chi_{a b}$ and it was thus neglected in the final analysis. The inertial defects were calculated to be $0.0856 \mathrm{amu} \AA^{2}$ for $23 \mathrm{DFBN}$ and $0.0578 \mathrm{amu}$ $\AA^{2}$ for $24 \mathrm{DFBN}$.

\section{Structure determination}

From the spectroscopic analysis, it was determined that the inertial defects of $\mathrm{BN}$ and its fluorinated derivatives are small and range from $-0.00111 \mathrm{amu} \AA^{2}$ to $0.117 \mathrm{amu} \AA^{2}$. Consequently, planarity was assumed in subsequent structural analysis.

i) $r_{e}$ equilibrium geometry

The geometry of each of the six compounds was optimized at the MP2 level using the basis set $6-311++\mathrm{G}(2 \mathrm{~d}, 2 \mathrm{p}))$ in Gaussian $09 .{ }^{40}$ Those related to the benzene ring and nitrile group are provided in Table 7 under the $\mathrm{r}_{\mathrm{e}}$ heading. Parameters involving $\mathrm{H}$ and $\mathrm{F}$ were needed to perform the subsequent structural analysis (as described below) and these are provided as Supporting Information. A natural bond orbital (NBO) analysis ${ }^{41}$ was performed for the optimized structures using the keyword $\mathrm{POP}=\mathrm{NBOREAD}$ in order to relate differences in the equilibrium geometry to specific changes to the electronic structure upon fluorination.

ii) $r_{s}$ substitution geometry

The rotational constants from each parent molecule and its heavy atom counterparts were 
used to perform a Kraitchman analysis ${ }^{42}$ to determine the corresponding atomic coordinates using the KRA program. ${ }^{43}$ It is important to note that the Kraitchman equations provide only absolute values of the coordinates and thus, the signs are inferred based on the orientation of each molecule in its principal axis system based on the ab initio predictions. The resulting $\mathrm{r}_{\mathrm{s}}$ parameters for BN, PFBN, 2FBN, 3FBN, 23DFBN and 24DFBN are summarized in Table 7. The uncertainties are calculated according to the method described by Costain ${ }^{44}$ which yields large errors in coordinates for atoms situated near a principal inertial axis. As depicted in Figure 1, this leads greater uncertainty in parameters involving certain atoms such as $\mathrm{C} 2$ in $2 \mathrm{FBN}$, $3 \mathrm{FBN}$ and 23DFBN and C6 in 24DFBN.

iii) ro ground state geometry

The $r_{0}$ geometries of the six rings were obtained by fitting the experimentally determined rotational constants of each species to key geometric parameters that using the STRFIT program. ${ }^{43}$ In order to preserve the position of the $\mathrm{H}$ and $\mathrm{F}$ atoms relative to the rest of the ring, the C-F and H-F distances were fixed at the appropriate ab initio values as were the differences of the angles external to the ring $(\angle \mathrm{FC} 2 \mathrm{C} 3-\angle \mathrm{FC} 2 \mathrm{C} 1$, etc. $)$. For $2 \mathrm{FBN}$, for example, seven bond lengths (C7-N, C1-C7, C1-C2, C2-C3, C3-C4, C4-C5, C5-C6), two angles involving the cyano-substituent ( $\angle \mathrm{NC} 7 \mathrm{C} 1, \angle \mathrm{C} 7 \mathrm{C} 1 \mathrm{C} 2$ ) and four differences of angles (involving the $\mathrm{F}-\mathrm{C} 2$ bond and the $\mathrm{C}-\mathrm{H}$ bonds with $\mathrm{C} 3, \mathrm{C} 4, \mathrm{C} 5)$ were fit to determine the $\mathrm{BN}$ backbone geometry. The remaining external angles involving the $\mathrm{C} 6-\mathrm{H}$ bond, were then calculated trigonometrically from the fit parameters (considering the ab initio difference $(\angle \mathrm{HC} 6 \mathrm{C} 5-\angle \mathrm{HC} 6 \mathrm{C} 1)$ and the fit was repeated until the results no longer varied. A similar procedure was followed for the others taking symmetry into account for PFBN. The parameters and associated uncertainties for the 
aromatic ring and the cyano substituent as summarized in Table 7 under the headings $\mathrm{r}_{0}$. The maximum discrepancy between the observed and calculated rotational constants from this least squares fitting procedure was only $0.02 \%$.

\section{E. ${ }^{14} \mathrm{~N}$ hyperfine analysis}

Comparison of the derived nitrogen nuclear quadrupole coupling constants $(\chi)$ of the substituted BN molecules provides further insight regarding the local electric field gradient around the nitrogen nuclei across all species studied. The hyperfine constants reported in Tables 1-6 are in the molecular principal inertial frame although comparison of the field gradient along the bonding axis of the $\mathrm{N}$ atom would be more meaningful. For BN and PFBN, the inertial frame also coincides with the principal quadrupole tensor frame due to the symmetry. For the other species in this work, due to the near coincidence of the principal quadrupole tensor axis with the $\mathrm{C} \equiv \mathrm{N}$ bond, the quadrupole coupling constants along the bond may be obtained by diagonalizing the quadrupole coupling tensor matrix in the molecular inertial frame $(a, b, c)$, to obtain coupling constants in the quadrupole tensor frame $(\mathrm{x}, \mathrm{y}, \mathrm{z})$. The rotation angles between the two frames (a $\rightarrow \mathrm{z}, \mathrm{b} \rightarrow \mathrm{x}, \mathrm{c} \rightarrow \mathrm{y})$ are given in Table 8.

Unfortunately, the off-diagonal ${ }^{14} \mathrm{~N}$ nuclear quadrupole coupling constants $\left(\chi_{\mathrm{ab}}\right)$ of all species except 3FBN were not well-determined. For these cases, by assuming symmetric $\mathrm{C} \equiv \mathrm{N}$ bonding, i.e. symmetric field gradient around the nitrogen nucleus, a simple transformation can be done to first estimate the $\chi_{\text {bond }}\left(\right.$ also $\left.\chi_{z z}\right):{ }^{45}$

$$
\chi_{\mathrm{aa}}=\chi_{\mathrm{bond}} \times\left(3 \cos ^{2} \theta-1\right) / 2
$$

where $\chi_{\mathrm{aa}}$ is the nuclear quadrupole coupling constant along the least principal inertial axis; $\chi_{\text {bond }}$ is the coupling constant along the $\mathrm{C} \equiv \mathrm{N}$ bond and $\theta$ is the angle between the $\mathrm{C} \equiv \mathrm{N}$ bond and the 
least principal axis. For 3FBN (off-diagonal $\chi_{\mathrm{ab}}$ well determined), both methods provide virtually the same $\chi_{\text {bond }}$ values $(-4.26 \mathrm{MHz}$ vs. $-4.27 \mathrm{MHz}$ in Table 8$)$. In order to evaluate $\chi_{\mathrm{xx}}\left(\chi_{\mathrm{cc}}=\chi_{\mathrm{yy}}\right.$ due to the near coincidence of the $\mathrm{c}$ and $\mathrm{y}$ axis), one can use the equation

$$
\chi_{z z}+\chi_{x x}=\chi_{a a}+\chi_{b b}
$$

as the trace of the coupling tensor matrix should not change before and after the diagonalization. For 3FBN, both methods provide virtually the same $\chi_{\mathrm{xx}}$ values $(2.27 \mathrm{MHz}$ vs. $2.28 \mathrm{MHz}$ along with $\left.\chi_{\mathrm{cc}}=1.99 \mathrm{MHz}\right)$.

For atomic ${ }^{14} \mathrm{~N}$, since the $2 \mathrm{p}$ orbitals are half filled and thus produce a spherical field gradient around the nucleus, quadrupole coupling does not happen in the free atom. However, during bonding with other atoms, the hybridization of $\mathrm{p}$ atomic orbitals with s orbitals can cause the spherical field gradient of the p orbitals to become unbalanced and thus introduces significant electric coupling in molecules. For a $\mathrm{C} \equiv \mathrm{N}$ triple bond as in the $\mathrm{BN}$ molecules, the $\mathrm{p}_{\mathrm{z}}$ orbital of the $\mathrm{N}$ atom participates in sp hybridization to form one $\sigma$ bond with the carbon atom while the pure $\mathrm{p}_{\mathrm{x}}$ and $\mathrm{p}_{\mathrm{y}}$ orbitals form two $\pi$ bonds with carbon in - and normal to - the molecular plane, respectively. The model to interpret nuclear quadrupole coupling constants is well-described, ${ }^{46}$ taking the orbital hybridization, the covalent bonding polarity as well as the nuclear screening effect into account:

$$
\begin{aligned}
& \chi_{\mathrm{zz}}=\left[\left(\mathrm{n}_{2 \mathrm{p}_{\mathrm{z}}}-\frac{1}{2}\left(\mathrm{n}_{2 \mathrm{p}_{\mathrm{x}}}+\mathrm{n}_{2 \mathrm{p}_{\mathrm{y}}}\right)\right] \times \frac{e Q q_{210}(\mathrm{~N})}{1+\mathrm{c}^{-} \varepsilon_{\mathrm{N}}}\right. \\
& \chi_{\mathrm{xx}}-\chi_{\mathrm{yy}}=\frac{3}{2}\left(\mathrm{n}_{2 \mathrm{p}_{\mathrm{x}}}-\mathrm{n}_{2 \mathrm{p}_{\mathrm{y}}}\right) \times \frac{e Q q_{210}(\mathrm{~N})}{1+\mathrm{c}^{-} \varepsilon_{\mathrm{N}}}
\end{aligned}
$$

where $\mathrm{n}$ represents the number of electrons in the specified $\mathrm{p}$ orbitals; $\mathrm{eQq}_{210}(\mathrm{~N})$ is the free nitrogen atomic coupling constant of the $2 \mathrm{p}_{\mathrm{z}}$ electron with a value of $-10 \mathrm{MHz} ;\left(1+\mathrm{c}^{-} \varepsilon_{\mathrm{N}}\right)$ is the charge screening effect correction factor with $\mathrm{c}^{-}$, the negative charge on the $\mathrm{N}$ atom; $\varepsilon_{\mathrm{N}}$ is the 
nuclear screening effect constant, with a value of 0.3 for the nitrogen nucleus. According to Gordy and Cook, ${ }^{46}$ the electron distribution due to sp hybridization can be described as:

$$
\begin{aligned}
& \mathrm{n}_{2 \mathrm{p}_{\mathrm{z}}}=2 \alpha_{\mathrm{s}}^{2}+\left(1+\mathrm{i}_{\sigma}\right) \times\left(1-\alpha_{\mathrm{s}}^{2}\right) \\
& \mathrm{n}_{2 \mathrm{p}_{\mathrm{x}}}=1+\pi_{\mathrm{x}} \\
& \mathrm{n}_{2 \mathrm{p}_{\mathrm{y}}}=1+\pi_{\mathrm{y}}
\end{aligned}
$$

where $\alpha_{s}^{2}$ is the $s$ character in the hybrid orbitals and is assumed to be 0.5 for sp hybridization ${ }^{4}$; the ionic character $i_{\sigma}$ across the $\mathrm{C}-\mathrm{N}$ p $\sigma$ bond can be evaluated by the electronegativity difference between the carbon atom and nitrogen atom: $\mathrm{i}_{\sigma}=\left|\mathrm{x}_{\mathrm{C}}-\mathrm{x}_{\mathrm{N}}\right| / 2=0.25 ; \pi_{\mathrm{x}}$ and $\pi_{\mathrm{y}}$ are the ionic character across the $\mathrm{C}-\mathrm{N} \mathrm{p} \pi$ bonds in - and normal to - the molecular plane, respectively and the negative charge on the $N$ atom is $c^{-}=i_{\sigma}+\pi_{x}+\pi_{y}=i_{\sigma}+\pi_{c}$. Substituting equations (5)-(7) into (3) and (4), we obtain:

$$
\begin{aligned}
& \chi_{\text {bond }}=\left\{\left[\left(1+\mathrm{i}_{\sigma}\right)\left(1-\alpha_{\mathrm{s}}^{2}\right)+2 \alpha_{\mathrm{s}}^{2}\right]-\left(2+\pi_{\mathrm{x}}+\pi_{\mathrm{y}}\right) / 2\right\} \times \mathrm{eQq}_{210}(\mathrm{~N}) /\left[1+\left(\mathrm{i}_{\sigma}+\pi_{\mathrm{x}}+\pi_{\mathrm{y}}\right) \times \varepsilon_{\mathrm{N}}\right] \\
& \chi_{\mathrm{xx}}-\chi_{\mathrm{yy}}=\frac{3}{2}\left(\pi_{\mathrm{x}}-\pi_{\mathrm{y}}\right) \times \mathrm{eQq}_{210}(\mathrm{~N}) /\left[1+\left(\mathrm{i}_{\sigma}+\pi_{\mathrm{x}}+\pi_{\mathrm{y}}\right) \times \varepsilon_{\mathrm{N}}\right]
\end{aligned}
$$

For all species under study here, if we assume that the C-N po bonding is not affected by fluorine substitution, $\mathrm{i}_{\sigma}(0.25)$ and $\alpha_{s}^{2}(0.5)$ can be fixed for all species allowing equations (8) and (9) to be solved for the unknown $\pi_{\mathrm{x}}$ and $\pi_{\mathrm{y}}$. The resulting $\pi$ parameters are listed in Table 8 . The assumption of no perturbation of the $\sigma$ bond of $\mathrm{CN}$ is supported by results from $\mathrm{NBO}$ analysis of the optimized geometries which show only very minor changes to the character of the $\mathrm{C}-\mathrm{N}$ p $\sigma$ orbital as fluorine atoms are added to the ring. For $\mathrm{BN}$, for example, the NBO that describes the $\sigma$ bond of $\mathrm{CN}$ is $57.05 \%$ on nitrogen (the more electronegative of the two atoms) whereas in PFBN, the NBO is only slightly less concentrated on nitrogen (56.76\%); likely due to inductive effects from the more electronegative pentafluorobenezene ring. For the partially fluorinated species, the NBO character is intermediate to these two cases. 


\section{F. Discussion}

Comparison of the $\mathrm{r}_{0}$ and $\mathrm{r}_{\mathrm{e}}$ structures in Table 7 for the six molecules under study shows comparable trends and that the values themselves generally agree within the derived experimental uncertainties. The largest discrepancy occurs in the parameters involving atoms in close proximity to the $\mathrm{CN}$ group such as the $\mathrm{C} 1-\mathrm{C} 7$ and $\mathrm{C} 7-\mathrm{N}$ bond lengths as described in Figure 1. The C7-N bond length is predicted to remain constant at $1.173 \AA\left(\mathrm{r}_{\mathrm{e}}\right)$ regardless of the number of fluorine substituents and this is also observed in the experimentally-derived $\mathrm{r}_{0}$ structures, however, the values are smaller (1.156-1.158(3) $\AA$ ). The C1-C7 bond is more variable in length but the experimental values $\left(\mathrm{r}_{0}\right)$ tend to be $0.010-0.015 \AA$ greater than the $\mathrm{r}_{\mathrm{e}}$ values. Interestingly, as one parameter seems systematically overestimated and the other underestimated, the $\mathrm{C} 1-\mathrm{N}$ distance is close to the value expected at the MP2 level of theory. The reliability of the $r_{e}$ structures was checked by performing new calculations using DFT theory (B3LYP) with various basis sets and similar discrepancies were noted. Molecular dynamics (MD) simulations provide a possible explanation for the difficulty in modelling this seemingly simple molecule. ${ }^{11}$ The low frequency in-plane $\left(158-167 \mathrm{~cm}^{-1}\right)$ and out-of-plane $\left(141 \mathrm{~cm}^{-1}\right)$ bending modes in which the $\mathrm{C} 1 \mathrm{C} 7 \mathrm{~N}$ subunit wags about $\mathrm{C} 1$ led to an average structure that is nonplanar. In fact, MD simulations predict that the $\mathrm{C} 1 \mathrm{C} 7 \mathrm{~N}$ entity makes an angle of $8^{\circ}$ on average with the ring. When this was considered in the analysis of gas phase electron diffraction (GED) experiments of $\mathrm{BN},{ }^{11}$ the derived structure had a $\mathrm{C} 1 \mathrm{C} 7$ bond length of $1.4326(11) \AA$ (in agreement with the $\mathrm{r}_{\mathrm{e}}$ value in Table 7) and a C7N bond length of 1.1582(5) $\AA$ (in agreement with the $\mathrm{r}_{0}$ value in Table 7). A more recent theoretical study of $\mathrm{BN}$, however, found close agreement in the geometry derived from high level quantum chemical calculations (MP2 and $\operatorname{CCSD}(\mathrm{T})$ with 
quintuple-zeta basis sets) $\left(\mathrm{re}_{\mathrm{e}}^{\mathrm{BO}}\right)$ with that from semi-experimental calculations $\left(\mathrm{r}_{\mathrm{e}} \mathrm{SE}^{\mathrm{SE}}\right)$ which use experimental rotational constants combined with ab initio estimates of rovibrational corrections (that would presumably account for the vibrational averaging highlighted by the MD study). ${ }^{12}$ Although the $r_{e}$ parameters in Table 7 (this work) do not provide the most accurate geometry of BN around the cyano substituent, they provide a convenient means to track the anticipated changes that accompany fluorination in the other species under study here. The trends observed in the $r_{e}$ values appear to be mirrored in the $r_{0}$ parameters, as described below, that were derived from the microwave spectra.

\section{i) Monofluorobenzonitrile}

The geometric changes following fluorine substitution at the ortho or meta positions of the ring relative to $\mathrm{CN}$ can be observed through comparison of the $\mathrm{r}_{0}$ structures for $2 \mathrm{FBN}$ and $3 \mathrm{FBN}$ with that of $\mathrm{BN}$ in Table 7. In $2 \mathrm{FBN}$, the $\mathrm{C} 1 \mathrm{C} 2 \mathrm{C} 3$ ring angle opens by $\sim 3^{\circ}$ and the $\mathrm{C} 1-\mathrm{C} 2$ and C2-C3 bonds shorten by $0.013-0.014 \AA$ which mirrors the trends in the $\mathrm{r}_{\mathrm{e}}$ parameters. For $3 \mathrm{FBN}$, the same alterations are seen with the $\mathrm{C} 2 \mathrm{C} 3 \mathrm{C} 4$ angle opening by $\sim 4^{\circ}$ but the bond length change predicted for $\mathrm{C} 2-\mathrm{C} 3(0.007 \AA)$ is smaller than the experimental uncertainties in this parameter. These geometry changes are attributed to the inductive effects of the fluorine atom which draws electron density toward it creating a more polar C-F bond. Using Bent's rule,${ }^{47}$ the p-character of the hybrid orbital along this bond increases while the hybrid orbitals forming $\sigma$ bonds within the ring have less p-character (more s-character) leading to larger ring angles and shorter C-C bonds. These observations follow the trends observed in the related fluoropyridine ${ }^{8,9}$ and fluorobenzene ${ }^{6,7}$ rings and are in agreement with the NBO analysis which shows an increase in p-character of $\sim 6 \%$ along the $\mathrm{CF}$ bond in both $2 \mathrm{FBN}$ and $3 \mathrm{FBN}$. 
Differences in geometry around the $\mathrm{CN}$ substituent itself provide more insight into electronic structure changes that accompany fluorination. Comparison of the $\mathrm{C} 6 \mathrm{C} 1 \mathrm{C} 2$ angle that describes the ipso carbon of the $\mathrm{CN}$ substituent shows that this angle is reduced by $\sim 2^{\circ}$ in $2 \mathrm{FBN}$ relative to $\mathrm{BN}$ and $3 \mathrm{FBN}$. The smaller angle in $2 \mathrm{FBN}$ moves the fluorine and nitrogen atoms further apart and thus may be a means to compensate for electrostatic repulsion between them as these sites carry the greatest negative natural charge (F: $-0.384, \mathrm{~N}:-0.344)$. Comparison of the $\mathrm{NC7C1}$ angle (which is linear in $\mathrm{BN}$ ) reveals that when fluorine is ortho to $\mathrm{CN}$, the nitrogen atom is tilted slightly away from fluorine by $0.8(3)^{\circ}$; an effect not seen in $3 \mathrm{FBN}$. As part of the NBO analysis, second-order perturbation corrections to the energy were calculated to identify possible interactions between donor and acceptor orbitals. The results show that there is a potential stabilization $(0.65 \mathrm{kcal} / \mathrm{mol})$ through donation of electron density from the lone pair (n) on fluorine to the antibonding $\pi^{*}$ orbital of $\mathrm{CN}$ which would distort the linear geometry at $\mathrm{C} 7$ in 2FBN but not for 3FBN. This type of distortion arising from a through-space interaction between fluorine and $\mathrm{CN}$ has been observed before via x-ray crystallography and solution NMR in intramolecular systems. ${ }^{48}$

The ${ }^{14} \mathrm{~N}$ hyperfine splittings serve as an additional probe of the electronic structure on the nitrogen atom including the relative electron density available for $\pi$ bonding $\left(\pi_{\mathrm{c}}\right)$ and how this is partitioned between the in-plane $2 \mathrm{p}_{\mathrm{x}}$ orbital $\left(\pi_{\mathrm{x}}\right)$ versus out-of-plane $2 \mathrm{p}_{\mathrm{y}}$ orbital $\left(\pi_{\mathrm{y}}\right)$ in the various compounds as shown in Table 8 . The notation used by Gordy and $\operatorname{Cook}^{46}\left(\pi_{\mathrm{x}}\right.$ and $\left.\pi_{\mathrm{y}}\right)$ refers to the local electron density around nitrogen that contribute to the $\pi$ ionic character and should not be confused with the molecular orbitals delocalized between $\mathrm{C} 7$ and nitrogen. Compared with $\mathrm{BN}$ itself, the total charge on nitrogen $\left(c^{-}=i_{\sigma}+\pi_{\mathrm{x}}+\pi_{\mathrm{y}}=i_{\sigma}+\pi_{\mathrm{c}}\right)$ is slightly reduced in the monfluorinated species which is in agreement with the natural charges at this site from the NBO 
calculation: $\mathrm{BN}(-0.365), 2 \mathrm{FBN}(-0.344)$ and $3 \mathrm{FBN}(-0.349)$. The $\pi_{\mathrm{x}}$ character of $2 \mathrm{FBN}$ is reduced slightly relative to $\mathrm{BN}$ and $3 \mathrm{FBN}$ which is consistent with the NBO results that shows the $\pi_{\mathrm{x}}$ character on nitrogen in $2 \mathrm{FBN}$ is decreased by $0.53 \%$. This may be a reflection of the minor contributions from cumulenic $\mathrm{C}=\mathrm{C}=\mathrm{N}$ resonance structures caused by hyperconjugation of the $2 p_{y}$ lone pair of electrons on the ortho fluorine as shown in Figure 4. In such structures, the $\pi$ molecular orbital in $\mathrm{C}=\mathrm{N}$ is perpendicular to the $\pi$ system of the aromatic ring and its electron density is more evenly shared between the two centers which reduces the $\pi_{\mathrm{x}}$ ionic character at nitrogen itself. In comparison, the same resonance structures are not as favourable in $3 \mathrm{FBN}$ as shown in Figure 4 as the stabilization of the aromatic ring electrons is reduced. In the $\pi_{\mathrm{y}}$ orbitals on nitrogen, which are orientated to allow overlap with the aromatic ring, the electron density of is slightly depleted relative to $\mathrm{BN}$ which is mirrored in the NBO predictions of $0.38 \%$ and $0.48 \%$ reductions for $2 \mathrm{FBN}$ and $3 \mathrm{FBN}$, respectively. This may be due to the increased electronegativity of the $-\mathrm{C}_{6} \mathrm{H}_{4} \mathrm{~F}$ group as a whole relative to $-\mathrm{C}_{6} \mathrm{H}_{5}$.

As a final note, the ${ }^{14} \mathrm{~N}$ nuclear quadrupole coupling constants can be directly used to predict whether there are changes to the bonding of the $\mathrm{CN}$ substituent with fluorine substitution at various sites as described by Böttcher and Sutter. ${ }^{28}$ Using a LCAO-MO treatment, Böttcher and Sutter derived $\chi$ values in the quadrupole tensor frame showing that for nitrile-type bonding $(C-C \equiv N), \chi_{x x}=\chi_{y y}$ on nitrogen while for cumulenic arrangements $(C=C=N)$ arising from fluorine hyperconjugation, $\chi_{\mathrm{xx}}=-\chi_{\mathrm{yy}}$ on nitrogen (reported using the axis system in this work which is different than the one used in reference 28 ). Thus, $\chi_{\mathrm{xx}}-\chi_{\mathrm{yy}}$ provides a clue regarding the relative importance of these resonance structures for the various compounds. The results in Table 8 suggest that the nitrile and cumulenic resonance contributions are similar in BN 
and $2 \mathrm{FBN}$ but for $3 \mathrm{FBN}, \mathrm{C}=\mathrm{C}=\mathrm{N}$ type contributors are of reduced importance (Figure 4 ) in comparison. In fact, its value is smaller than for all other compounds studied here.

\section{ii) Difluorobenzonitrile}

Changes to the ring geometry in 23DFBN and 24DFBN follow those reported earlier for the difluoropyridines ${ }^{9}$ and are consistent with the trends in the $r_{e}$ parameters. As described above, the inductive effect of fluorine tends to open the ring angle at the site of fluorination and the C-C ring bonds involving the ipso carbon tend to shorten although the uncertainties in $\mathrm{r}_{0}$ values for bond lengths are too large in some cases to confirm this. When substitution occurs on two adjacent carbons, as in 23DFBN (and 2,3-difluoropyridine (DFP)), the angle opening is muted at each fluorination site $\left(\sim 1^{\circ}\right.$ for $23 \mathrm{DFBN}, 0.4-0.7^{\circ}$ for $\left.23 \mathrm{DFP}\right)$ compared to the reference compound to balance the inductive effect of fluorine (the tendency to widen the angle) with the destabilizing effect of increasing ring strain at other angles. Concerning the geometry close to the $\mathrm{CN}$ substituent, the $\mathrm{C} 6 \mathrm{C} 1 \mathrm{C} 2$ angle in both $23 \mathrm{DFBN}$ and $24 \mathrm{DFBN}$ is $\sim 2^{\circ}$ smaller relative to that of $\mathrm{BN}$ as seen in $2 \mathrm{FBN}$. For the $\mathrm{NC} 7 \mathrm{C} 1$ angles, the $\mathrm{r}_{\mathrm{e}}$ values predict deviation from linearity as in $2 \mathrm{FBN}$ and the NBO analysis shows a similar degree of $\mathrm{n} \rightarrow \pi^{*}$ donation $(0.61-0.63 \mathrm{kcal} / \mathrm{mol})$.

As in the monofluorinated species, the total charge on nitrogen is less in 23DFBN (-0.330) and 24DFBN (-0.340) relative to BN $(-0.360)$ and is largely a consequence of changes to the $\pi_{\mathrm{x}}$ and $\pi_{\mathrm{y}}$ ionic character on nitrogen. The trend in Table 8 shows a reduction in both $\pi_{\mathrm{x}}$ and $\pi_{\mathrm{y}}$ relative to $\mathrm{BN}$. These observations are consistent with the NBO calculations showing $0.67 \%$ and $0.88 \%$ decreases in the $\pi_{\mathrm{x}}$ orbital at nitrogen in $23 \mathrm{DFBN}$ and $24 \mathrm{DFBN}$, respectively due to more delocalization of the electron density across $\mathrm{C} 7$ and nitrogen in the cumulenic contributors. There is a $0.81 \%$ reduction for $\pi_{\mathrm{y}}$ on nitrogen in $23 \mathrm{DFBN}$ and a smaller reduction in $24 \mathrm{DFBN}$ 
$(0.24 \%)$. If one considers resonance structures based on donation of the lone pair on fluorine to the $\pi$ system of the aromatic ring, both 23DFBN and 24DFBN have cumulenic contributors using the ortho fluorine substituent but the latter will have additional structures of this type from the para fluorine. This will maintain greater electron density in $\pi_{\mathrm{y}}$ on nitrogen that partially counteracts the electron withdrawing effect of the increased electronegativity of the $-\mathrm{C}_{6} \mathrm{H}_{3} \mathrm{~F}_{2}$ group. The added importance of these cumulenic resonance contributors in 24DFBN is supported by the greater negative charge on nitrogen in 24DFBN $(-0.459)$ relative to 23DFBN $(-0.326)$ and by the values of $\chi_{\mathrm{xx}}-\chi_{\mathrm{yy}}$ in Table 8 .

\section{iii) Pentafluorobenzonitrile}

Comparison of the $\mathrm{r}_{0}$ structures for $\mathrm{BN}$ and PFBN in Table 7 shows several interesting geometry changes. Upon perfluorination, the ring contracts as all bond lengths are reduced via inductive effects of the fluorine atoms. The change is most significant for $\mathrm{C} 2-\mathrm{C} 3$ which may suggest that resonance structures in which the para fluorine atom donates electron density to the ring through hyperconjugation are favoured over other structures of this type. This is consistent with the increased charge on $\mathrm{C} 1$ in PFBN (-0.294) versus BN (-0.198). Finally, the C6C1C2 angle is reduced by $\sim 3^{\circ}$ as reported above for the species with ortho fluorine atoms.

The charge at nitrogen (-0.293) of PFBN is the smallest of the six compounds studied which is as expected due to the group electronegativity of $-\mathrm{C}_{6} \mathrm{~F}_{5}$. This is reflected in the ${ }^{14} \mathrm{~N}$ hyperfine analysis giving the lowest electron density in $\pi_{\mathrm{c}}=\pi_{\mathrm{x}}+\pi_{\mathrm{y}}$ at nitrogen in Table 8 . Compared to BN, both the $\pi_{\mathrm{x}}$ and $\pi_{\mathrm{y}}$ ionic character is reduced for PFBN and this is consistent with the NBO analysis which shows $1.7 \%$ and $1.4 \%$ decrease in electron density on these nitrogen orbitals compared to the reference compound. As in the previous cases, this reflects the 
importance of nitrile versus cumulenic-type resonance contributions that change the electronic environment at nitrogen. Compared with the mono- and difluorinated rings, NBO results show that the $\sigma \mathrm{CN}$ bond is more affected in PFBN than for the other compounds with a $0.29 \%$ reduction in electron density on nitrogen for this orbital relative to that of BN. This suggests that the inductive effects from the five electron withdrawing fluorine atoms extend beyond the $\mathrm{C} 1 \mathrm{C} 7$ bond in this case. Finally, the $\chi_{x x}-\chi_{y y}$ value of PFBN in Table 8 shows that it is intermediate to those of 23DFBN and 24DFBN which fits with mesomeric arguments for the three ortho/para substituents (favouring an increase in cumulenic contributions) versus the two meta fluorine atoms (enhancing the nitrile character).

In conclusion, we measured and assigned the pure rotational spectra of the normal species of BN, PFBN, 2FBN, 3FBN, 23DFBN and 24DFBN and their ${ }^{13} \mathrm{C}$ and ${ }^{15} \mathrm{~N}$ substituted analogues. Analysis of the spectra provided estimates of the heavy atom geometries and the trends observed compare favourably with ab initio results at the MP2 level $(6-311++\mathrm{G}(2 \mathrm{~d}, 2 \mathrm{p}))$. As seen for other fluorine substituted aromatic rings, the ring angle at the site of fluorination increases and the adjacent $\mathrm{C}-\mathrm{C}$ bonds decrease. When one fluorine atom is ortho to $\mathrm{CN}$, the ring angle at $\mathrm{C} 1$ decreases and the $\mathrm{CN}$ bond tilts away from fluorine. These trends are explained using a combination of electrostatic, inductive and resonance arguments and are supported by NBO calculations as well as information derived from the observed ${ }^{14} \mathrm{~N}$ hyperfine structure.

\section{Acknowledgements}

This research is funded by the Natural Sciences and Engineering Research Council of Canada (NSERC) through a Discovery Grant awarded to JvW.

\section{Supporting information}


Assigned transition frequencies for the six compounds (Appendix 1-6). Ab initio geometry parameters (Appendix 7). This material is available free of charge via the Internet at http://pubs.acs.org. 
Table 1: Ground state spectroscopic constants of benzonitrile $(\mathrm{BN})$ and its isotopologues (A reduction, $\mathrm{I}^{\mathrm{r}}$ representation).

\begin{tabular}{|c|c|c|c|c|c|c|c|}
\hline & Normal & ${ }^{13} \mathrm{C} 1$ & ${ }^{13} \mathrm{C} 2$ & ${ }^{13} \mathrm{C} 3$ & ${ }^{13} \mathrm{C} 4$ & ${ }^{13} \mathrm{C} 7$ & ${ }^{15} \mathrm{~N}$ \\
\hline \multicolumn{8}{|c|}{ Rotational constants ${ }^{\mathrm{a}, \mathrm{b}} / \mathrm{MHz}$} \\
\hline A & $5655.2653(22)$ & $5655.5075(55)$ & $5563.9185(45)$ & $5565.6669(50)$ & $5655.4544(75)$ & $5655.2407(31)$ & $5655.270(15)$ \\
\hline B & $1546.875787(64)$ & $1545.55183(16)$ & $1546.80340(13)$ & $1535.71305(14)$ & $1523.65523(16)$ & $1528.64068(16)$ & $1502.14915(29)$ \\
\hline $\mathrm{C}$ & $1214.404045(54)$ & $1213.60145(10)$ & $1210.089718(98)$ & $1203.37302(10)$ & $1200.05780(10)$ & $1203.136828(95)$ & $1186.65856(21)$ \\
\hline \multicolumn{8}{|c|}{${ }^{14} \mathrm{~N}$ nuclear quadrupole coupling constants ${ }^{\mathrm{c}} / \mathrm{MHz}$} \\
\hline $1.5\left(\chi_{\mathrm{aa}}\right)$ & $-6.35579(76)$ & $-6.3438(84)$ & $-6.3666(80)$ & $-6.3661(86)$ & $-6.345(14)$ & $-6.360(16)$ & \\
\hline $0.25\left(\chi_{\mathrm{bb}-}-\chi_{\mathrm{cc}}\right)$ & $0.08490(31)$ & $0.0724(51)$ & $0.0696(48)$ & $0.0681(52)$ & $0.0749(48)$ & $0.0758(53)$ & \\
\hline \multicolumn{8}{|c|}{ Centrifugal distortion constants ${ }^{\mathrm{d}} / \mathrm{kHz}$} \\
\hline$\Delta_{\mathrm{J}}$ & $0.04500(34)$ & $0.04616(77)$ & $0.04573(64)$ & $0.04510(69)$ & $0.0446(10)$ & $0.04513(86)$ & $0.0392(37)$ \\
\hline$\Delta_{\mathrm{IK}}$ & $0.9372(19)$ & $0.879(18)$ & $0.921(13)$ & $0.937(14)$ & $0.907(26)$ & $0.899(21)$ & $0.911(28)$ \\
\hline$\delta_{\mathrm{j}}$ & $0.01121(23)$ & $0.01111(72)$ & $0.01146(64)$ & $0.01103(70)$ & $0.01186(77)$ & $0.01057(74)$ & $0.0172(38)$ \\
\hline$\frac{j}{\delta}$ & $0.603(13)$ & 0.603 & 0.603 & 0.603 & 0.603 & 0.603 & 0.603 \\
\hline $\mathrm{rms} / \mathrm{kHz}$ & 1.9 & 2.1 & 2.1 & 2.3 & 1.8 & 2.0 & 0.8 \\
\hline \# lines & $188 a$-type & $51 a$-type & $63 a$-type & $63 a$-type & $42 a$-type & $39 a$-type & $11 a$-type \\
\hline
\end{tabular}

${ }^{a}$ Rotational constants from reference 19: $\mathrm{A}=5655.314(16) \mathrm{MHz}, \mathrm{B}=1546.8768(15) \mathrm{MHz}, \mathrm{C}=1214.4032(14) \mathrm{MHz}$

${ }^{\mathrm{b}}$ Calculated rotational constants $(\mathrm{MP} 2 / 6-311++\mathrm{G}(2 \mathrm{~d}, 2 \mathrm{p}))$ from this work: $\mathrm{A}=5666.8 \mathrm{MHz}, \mathrm{B}=1540.7 \mathrm{MHz}, \mathrm{C}=1211.4 \mathrm{MHz}$

${ }^{\mathrm{c}}$ Calculated ${ }^{14} \mathrm{~N}$ hyperfine constants from this work: B3LYP/6-311++G(2d,2p): $1.5\left(\chi_{\mathrm{aa}}\right)=-6.382 \mathrm{MHz}, 0.25\left(\chi_{\mathrm{bb}}-\chi_{\mathrm{cc}}\right)=0.095 \mathrm{MHz}$; $\mathrm{MP} 2 / 6-311++\mathrm{G}(2 \mathrm{~d}, 2 \mathrm{p}): 1.5\left(\chi_{\mathrm{aa}}\right)=-5.842 \mathrm{MHz}, 0.25\left(\chi_{\mathrm{bb}}-\chi_{\mathrm{cc}}\right)=0.018 \mathrm{MHz}$

${ }^{\mathrm{d}}$ Some centrifugal distortion constants for minor isotopologues were held fixed to the parent values during the fit. These are given here without uncertainties. 
Table 2: Ground state spectroscopic constants of pentafluorobenzonitrile (PFBN) and its isotopologues.

\begin{tabular}{|c|c|c|c|c|c|c|c|}
\hline & Normal & ${ }^{13} \mathrm{C} 1$ & ${ }^{13} \mathrm{C} 2$ & ${ }^{13} \mathrm{C} 3$ & ${ }^{13} \mathrm{C} 4$ & ${ }^{13} \mathrm{C} 7$ & ${ }^{15} \mathrm{~N}$ \\
\hline \multicolumn{8}{|c|}{ Rotational constants ${ }^{\mathrm{a}, \mathrm{b}} / \mathrm{MHz}$} \\
\hline A & $1029.368635(32)$ & $1029.40041(35)$ & $1026.38602(26)$ & $1026.36077(29)$ & $1029.40457(34)$ & $1029.36990(42)$ & $1029.3708(25)$ \\
\hline $\mathrm{B}$ & $764.5952880(91)$ & $762.92535(24)$ & $764.329754(66)$ & $763.68961(11)$ & $761.72244(24)$ & $756.63541(27)$ & $748.4102(14)$ \\
\hline $\mathrm{C}$ & $438.7218484(60)$ & $438.177629(29)$ & $438.092228(27)$ & $437.877120(26)$ & $437.781250(31)$ & $436.089678(33)$ & $433.343572(41)$ \\
\hline \multicolumn{8}{|c|}{${ }^{14} \mathrm{~N}$ nuclear quadrupole coupling constants ${ }^{\mathrm{c}} / \mathrm{MHz}$} \\
\hline $1.5\left(\chi_{\mathrm{aa}}\right)$ & $-6.5807(12)$ & $-6.583(39)$ & $-6.574(20)$ & $-6.577(23)$ & $-6.592(39)$ & $-6.625(45)$ & \\
\hline $0.25\left(\chi_{b b}-\chi_{c c}\right)$ & $0.10804(68)$ & $0.1055(29)$ & $0.1107(26)$ & $0.1082(26)$ & $0.1080(29)$ & $0.1080(34)$ & \\
\hline \multicolumn{8}{|c|}{ Centrifugal distortion constants $\mathrm{d} / \mathrm{kHz}$} \\
\hline$\Delta_{\mathrm{J}}$ & $0.006169(18)$ & $0.006096(92)$ & $0.006199(85)$ & $0.006150(84)$ & $0.006262(96)$ & $0.00616(10)$ & $0.00613(12)$ \\
\hline$\Delta_{\mathrm{JK}}$ & $0.044977(74)$ & $0.0478(23)$ & $0.0454(16)$ & $0.0468(16)$ & $0.0463(19)$ & $0.0453(27)$ & $0.0429(26)$ \\
\hline$\Delta_{\mathrm{K}}$ & $-0.02835(34)$ & $-0.02835^{\mathrm{d}}$ & -0.02835 & -0.02835 & -0.02835 & -0.02835 & -0.02835 \\
\hline$\delta_{\mathrm{i}}$ & $0.0023687(96)$ & 0.0023687 & 0.0023687 & 0.0023687 & 0.0023687 & 0.0023687 & 0.0023687 \\
\hline$\delta_{\mathrm{k}}$ & $0.029550(65)$ & 0.029550 & 0.029550 & 0.029550 & 0.029550 & 0.029550 & 0.029550 \\
\hline $\mathrm{rms} / \mathrm{kHz}$ & 1.5 & 2.3 & 2.2 & 2.3 & 2.3 & 2.5 & 1.5 \\
\hline \# lines & $753 a$-type & $81 a$-type & $99 a$-type & $96 a$-type & $81 a$-type & $78 a$-type & $30 a$-type \\
\hline
\end{tabular}

${ }^{a}$ Rotational constants from ref.25: $\mathrm{A}=1029.351(44) \mathrm{MHz}, \mathrm{B}=764.603(21) \mathrm{MHz}, \mathrm{C}=438.7215(33) \mathrm{MHz}$

${ }^{\mathrm{b}}$ Calculated rotational constants $(\mathrm{MP} 2 / 6-311++\mathrm{G}(2 \mathrm{~d}, 2 \mathrm{p}))$ from this work: $\mathrm{A}=1025.8 \mathrm{MHz}, \mathrm{B}=761.8 \mathrm{MHz}, \mathrm{C}=437.2 \mathrm{MHz}$

${ }^{\mathrm{c}}$ Calculated ${ }^{14} \mathrm{~N}$ hyperfine constants from this work: B3LYP/6-311++G(2d,2p): $1.5\left(\chi_{\mathrm{aa}}\right)=-6.623 \mathrm{MHz}, 0.25\left(\chi_{\mathrm{bb}}-\chi_{\mathrm{cc}}\right)=0.150 \mathrm{MHz}$; $\mathrm{MP} 2 / 6-311++\mathrm{G}(2 \mathrm{~d}, 2 \mathrm{p}): 1.5\left(\chi_{\mathrm{aa}}\right)=-5.988 \mathrm{MHz}, 0.25\left(\chi_{\mathrm{bb}}-\chi_{\mathrm{cc}}\right)=0.041 \mathrm{MHz}$

d Some centrifugal distortion constants for minor isotopologues were held fixed to the parent values during the fit. These are given here without uncertainties. 
Table 3: Ground state spectroscopic constants of 2-fluorobenzonitrile (2FBN) and its isotopologues.

\begin{tabular}{|c|c|c|c|c|c|c|c|c|c|}
\hline & Normal & ${ }^{13} \mathrm{C} 1$ & ${ }^{13} \mathrm{C} 2$ & ${ }^{13} \mathrm{C} 3$ & ${ }^{13} \mathrm{C} 4$ & ${ }^{13} \mathrm{C} 5$ & ${ }^{13} \mathrm{C} 6$ & ${ }^{13} \mathrm{C} 7$ & ${ }^{15} \mathrm{~N}$ \\
\hline \multicolumn{10}{|c|}{ Rotational constants ${ }^{\mathrm{a}, \mathrm{b}} / \mathrm{MHz}$} \\
\hline A & $2940.761152(88)$ & $2938.1093(17)$ & $2928.2761(24)$ & $2922.5534(20)$ & $2940.6938(25)$ & $2910.0201(16)$ & $2901.5861(19)$ & $2935.6537(17)$ & $2932.9825(14)$ \\
\hline $\mathrm{B}$ & $1512.700202(21)$ & $1511.90776(24)$ & $1512.65041(29)$ & $1502.35511(27)$ & $1488.44249(32)$ & $1498.29853(23)$ & $1511.98048(29)$ & $1497.25539(22)$ & $1473.13738(26)$ \\
\hline $\mathrm{C}$ & $998.652010(12)$ & $998.00280(13)$ & $997.18671(17)$ & $992.04545(16)$ & $988.01581(19)$ & $988.82807(13)$ & $993.78347(17)$ & $991.31529(14)$ & $980.37874(14)$ \\
\hline \multicolumn{10}{|c|}{${ }^{14} \mathrm{~N}$ nuclear quadrupole coupling constants ${ }^{\mathrm{c}} / \mathrm{MHz}$} \\
\hline $1.5\left(\chi_{\mathrm{aa}}\right)$ & $-6.27358(75)$ & $-6.2736(60)$ & $-6.289(10)$ & $-6.3029(85)$ & $-6.2776(92)$ & $-6.2329(58)$ & $-6.2621(73)$ & $-6.2879(62)$ & \\
\hline $0.25\left(\chi_{b b}-\chi_{c c}\right)$ & $0.06078(20)$ & $0.0572(23)$ & $0.0576(30)$ & $0.0612(28)$ & $0.0604(33)$ & $0.0557(24)$ & $0.0547(29)$ & $0.0618(22)$ & \\
\hline$\Delta_{\mathrm{J}}$ & $0.04051(15)$ & $0.0416(36)$ & $0.0431(45)$ & $0.0366(42)$ & $0.0404(46)$ & $0.0429(35)$ & $0.0435(45)$ & $0.0414(34)$ & $0.0416(24)$ \\
\hline$\Delta_{\mathrm{IK}}$ & $0.50832(80)$ & $0.506(26)$ & $0.526(35)$ & $0.550(32)$ & $0.473(35)$ & $0.477(25)$ & $0.484(33)$ & $0.510(26)$ & $0.496(18)$ \\
\hline$\Delta_{\mathrm{K}}$ & $0.4332(82)$ & 0.4332 & 0.4332 & 0.4332 & 0.4332 & 0.4332 & 0.4332 & 0.4332 & 0.4332 \\
\hline$\delta_{\mathrm{j}}$ & $0.012490(80)$ & $0.0135(23)$ & $0.0130(29)$ & $0.0093(27)$ & $0.0122(31)$ & $0.0131(23)$ & $0.0120(29)$ & $0.0137(22)$ & $0.0125(19)$ \\
\hline$\delta_{\mathrm{k}}$ & $0.3018(10)$ & 0.3018 & 0.3018 & 0.3018 & 0.3018 & 0.3018 & 0.3018 & 0.3018 & 0.3018 \\
\hline $\mathrm{rms} / \mathrm{kHz}$ & 2.1 & 1.9 & 2.3 & 2.3 & 2.5 & 1.8 & 2.3 & 1.9 & 0.8 \\
\hline \# lines & $291 a$-, $81 b$-type & $53 a$-type & $40 a$-type & $44 a$-type & $40 a$-type & $42 a$-type & $43 a$-type & $42 a$-type & $12 a$-type \\
\hline
\end{tabular}

a Rotational constants from reference 26: $\mathrm{A}=2940.745(12) \mathrm{MHz}, \mathrm{B}=1512.699(1) \mathrm{MHz}, \mathrm{C}=998.653(1) \mathrm{MHz}$

${ }^{\mathrm{b}}$ Calculated rotational constants $(\mathrm{MP} 2 / 6-311++\mathrm{G}(2 \mathrm{~d}, 2 \mathrm{p}))$ from this work: $\mathrm{A}=2932.6 \mathrm{MHz}, \mathrm{B}=1507.3 \mathrm{MHz}, \mathrm{C}=995.6 \mathrm{MHz}$

${ }^{\mathrm{c}}$ Calculated ${ }^{14} \mathrm{~N}$ hyperfine constants from this work: B3LYP/6-311++G(2d,2p): $1.5\left(\chi_{\mathrm{aa}}\right)=-6.303 \mathrm{MHz}, 0.25\left(\chi_{\mathrm{bb}}-\chi_{\mathrm{cc}}\right)=0.077 \mathrm{MHz}$, $\chi_{\mathrm{ab}}=-0.837 \mathrm{MHz} ; \mathrm{MP} 2 / 6-311++\mathrm{G}(2 \mathrm{~d}, 2 \mathrm{p}): 1.5\left(\chi_{\mathrm{aa}}\right)=-5.748 \mathrm{MHz}, 0.25\left(\chi_{\mathrm{bb}}-\chi_{\mathrm{cc}}\right)=-0.014 \mathrm{MHz}, \chi_{\mathrm{ab}}=-0.736 \mathrm{MHz}$.

d Some centrifugal distortion constants for minor isotopologues were held fixed to the parent values during the fit. These are given here without uncertainties. 
Table 4: Ground state spectroscopic constants of 3-fluorobenzonitrile (3FBN) and its isotopologues.

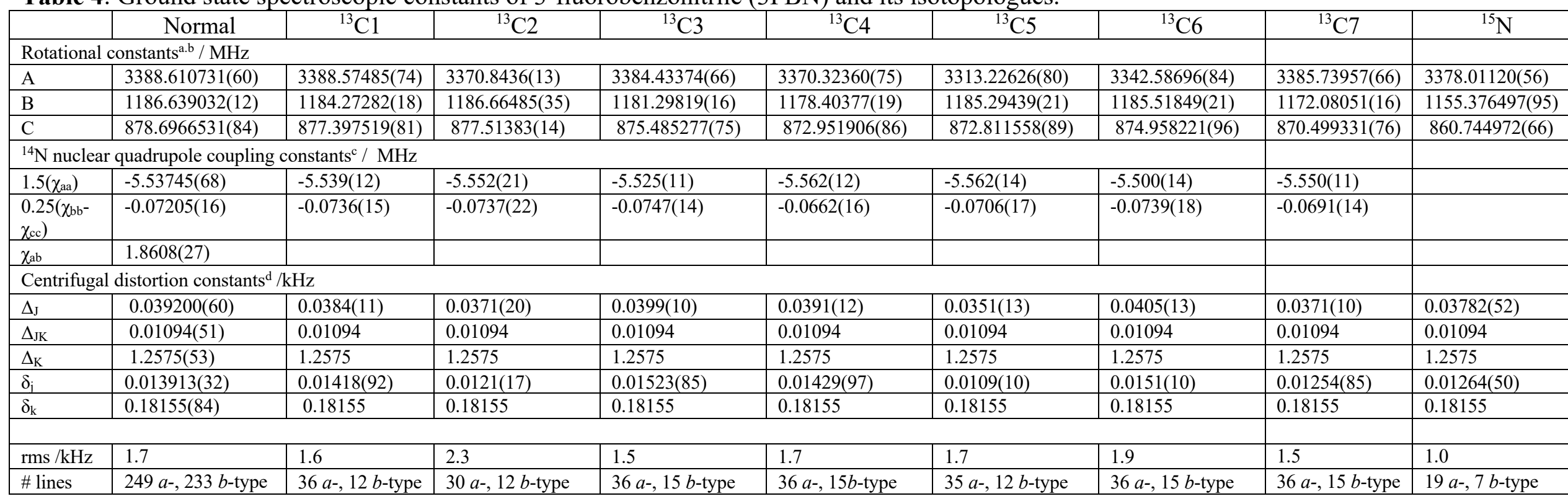

${ }^{a}$ Rotational constants from reference 28: $\mathrm{A}=3388.61073(35) \mathrm{MHz}, \mathrm{B}=1186.63868(13) \mathrm{MHz}, \mathrm{C}=878.69740(10) \mathrm{MHz}$

${ }^{\mathrm{b}}$ Calculated rotational constants $(\mathrm{MP} 2 / 6-311++\mathrm{G}(2 \mathrm{~d}, 2 \mathrm{p})$ ) from this work: $\mathrm{A}=3383.9 \mathrm{MHz}, \mathrm{B}=1181.8 \mathrm{MHz}, \mathrm{C}=875.9 \mathrm{MHz}$

${ }^{\mathrm{c}}$ Calculated ${ }^{14} \mathrm{~N}$ hyperfine constants from this work: B3LYP/6-311++G(2d,2p): $1.5\left(\chi_{\mathrm{aa}}\right)=-5.562 \mathrm{MHz}, 0.25\left(\chi_{\mathrm{bb}}-\chi_{\mathrm{cc}}\right)=-0.063 \mathrm{MHz}$, $\chi_{\mathrm{ab}}=1.875 \mathrm{MHz} ; \mathrm{MP} 2 / 6-311++\mathrm{G}(2 \mathrm{~d}, 2 \mathrm{p}): 1.5\left(\chi_{\mathrm{aa}}\right)=-5.066 \mathrm{MHz}, 0.25\left(\chi_{\mathrm{bb}}-\chi_{\mathrm{cc}}\right)=-0.099 \mathrm{MHz}, \chi_{\mathrm{ab}}=1.680 \mathrm{MHz}$.

${ }^{\mathrm{d}}$ Some centrifugal distortion constants for minor isotopologues were held fixed to the parent values during the fit. These are given here without uncertainties. 
Table 5: Ground state spectroscopic constants of 2,3-difluorobenzonitrile (23DFBN) and its isotopologues.

\begin{tabular}{|c|c|c|c|c|c|c|c|c|c|}
\hline & Normal & ${ }^{13} \mathrm{C} 1$ & ${ }^{13} \mathrm{C} 2$ & ${ }^{13} \mathrm{C} 3$ & ${ }^{13} \mathrm{C} 4$ & ${ }^{13} \mathrm{C} 5$ & ${ }^{13} \mathrm{C} 6$ & ${ }^{13} \mathrm{C} 7$ & ${ }^{15} \mathrm{~N}$ \\
\hline \multicolumn{10}{|c|}{ Rotational constants $^{\mathrm{a}, \mathrm{b}} / \mathrm{MHz}$} \\
\hline $\mathrm{A}$ & $2260.152021(47)$ & $2258.73587(84)$ & $2256.79214(88)$ & $2259.81277(73)$ & $2247.48304(89)$ & $2216.33857(72)$ & $2230.08374(72)$ & $2260.1463(10)$ & $2259.19885(36)$ \\
\hline $\mathrm{B}$ & $1182.866459(15)$ & $1180.64140(20)$ & $1182.89578(21)$ & $1177.28393(18)$ & $1174.06050(22)$ & $1181.13493(18)$ & $1182.01545(18)$ & $1168.65576(27)$ & $1151.97324(10)$ \\
\hline \multicolumn{10}{|c|}{${ }^{14} \mathrm{~N}$ nuclear quadrupole coupling constants ${ }^{\mathrm{c}} / \mathrm{MHz}$} \\
\hline $1.5\left(\chi_{\mathrm{aa}}\right)$ & $-5.80400(76)$ & $-5.7902(88)$ & $-5.8036(94)$ & $-5.7990(78)$ & $-5.8469(98)$ & $-5.8597(79)$ & $-5.7847(79)$ & $-5.815(12)$ & \\
\hline $\begin{array}{l}0.25\left(\chi_{\mathrm{bb}^{-}}\right. \\
\left.\chi_{\mathrm{cc}}\right)\end{array}$ & $-0.03156(20)$ & $-0.0339(16)$ & $-0.0311(14)$ & $-0.0332(13)$ & $-0.0235(17)$ & $-0.0235(13)$ & $-0.0355(13)$ & $-0.0330(20)$ & \\
\hline \multicolumn{10}{|c|}{ Centrifugal distortion constants ${ }^{\mathrm{d}} / \mathrm{kHz}$} \\
\hline$\Delta_{\mathrm{J}}$ & $0.034910(91)$ & $0.0359(16)$ & $0.0357(15)$ & $0.0346(14)$ & $0.0346(14)$ & $0.0331(14)$ & $0.0363(14)$ & $0.0357(21)$ & $0.03396(88)$ \\
\hline$\Delta_{\mathrm{IK}}$ & $0.17894(45)$ & $0.164(15)$ & $0.173(19)$ & $0.159(13)$ & $0.157(13)$ & $0.183(13)$ & $0.160(13)$ & $0.167(20)$ & $0.1802(58)$ \\
\hline$\Delta_{\mathrm{K}}$ & $0.0479(27)$ & 0.0479 & 0.0479 & 0.0479 & 0.0479 & 0.0479 & 0.0479 & 0.0479 & 0.0479 \\
\hline$\delta_{\mathrm{j}}$ & $0.012851(41)$ & $0.01320(88)$ & $0.01304(88)$ & $0.01229(78)$ & $0.01231(79)$ & $0.01179(79)$ & $0.01390(80)$ & $0.0135(12)$ & $0.01226(47)$ \\
\hline$\delta_{\mathrm{k}}$ & $0.16072(59)$ & $0.168(13)$ & $0.174(18)$ & $0.157(12)$ & $0.157(12)$ & $0.155(11)$ & $0.163(11)$ & $0.165(18)$ & $0.1627(69)$ \\
\hline $\mathrm{rms} / \mathrm{kHz}$ & 1.8 & 1.9 & 1.9 & 1.7 & 2.1 & 1.7 & 1.7 & 2.5 & 0.8 \\
\hline
\end{tabular}

${ }^{a}$ Rotational constants from reference 36: $\mathrm{A}=2260.1475(25) \mathrm{MHz}, \mathrm{B}=1182.8638(2) \mathrm{MHz}, \mathrm{C}=776.3842(1) \mathrm{MHz}$

${ }^{\mathrm{b}}$ Calculated rotational constants $(\mathrm{MP} 2 / 6-311++\mathrm{G}(2 \mathrm{~d}, 2 \mathrm{p}))$ from this work: $\mathrm{A}=2254.3 \mathrm{MHz}, \mathrm{B}=1178.9 \mathrm{MHz}, \mathrm{C}=774.1 \mathrm{MHz}$

${ }^{\mathrm{c}}$ Calculated ${ }^{14} \mathrm{~N}$ hyperfine constants from this work: B3LYP/6-311++G(2d,2p): $1.5\left(\chi_{\mathrm{aa}}\right)=-5.844 \mathrm{MHz}, 0.25\left(\chi_{\mathrm{bb}}-\chi_{\mathrm{cc}}\right)=-0.013 \mathrm{MHz}, \chi_{\mathrm{ab}}=$ $-1.679 \mathrm{MHz} ; \mathrm{MP} 2 / 6-311++\mathrm{G}(2 \mathrm{~d}, 2 \mathrm{p}): 1.5\left(\chi_{\mathrm{aa}}\right)=-5.312 \mathrm{MHz}, 0.25\left(\chi_{\mathrm{bb}}-\chi_{\mathrm{cc}}\right)=-0.076 \mathrm{MHz}, \chi_{\mathrm{ab}}=-1.496 \mathrm{MHz}$.

d Some centrifugal distortion constants for minor isotopologues were held fixed to the parent values during the fit. These are given here without uncertainties. 
Table 6: Ground state spectroscopic constants of 2,4-difluorobenzonitrile (24DFBN) and its isotopologues.

\begin{tabular}{|c|c|c|c|c|c|c|c|c|c|}
\hline & Normal & ${ }^{13} \mathrm{C} 1$ & ${ }^{13} \mathrm{C} 2$ & ${ }^{13} \mathrm{C} 3$ & ${ }^{13} \mathrm{C} 4$ & ${ }^{13} \mathrm{C} 5$ & ${ }^{13} \mathrm{C} 6$ & ${ }^{13} \mathrm{C} 7$ & ${ }^{15} \mathrm{~N}$ \\
\hline \multicolumn{10}{|c|}{ Rotational constants ${ }^{\mathrm{a}} / \mathrm{MHz}$} \\
\hline A & $2932.25895(36)$ & $2929.6350(30)$ & 2919.9804(22) & $2914.4250(32)$ & $2932.1085(37)$ & $2899.6703(29)$ & $2892.9047(31)$ & $2927.4453(32)$ & $2925.0155(15)$ \\
\hline $\mathrm{B}$ & $933.040760(27)$ & $931.64092(18)$ & $932.89099(15)$ & $931.14445(20)$ & $927.30253(22)$ & $930.13769(17)$ & $933.04662(19)$ & $923.75791(19)$ & $912.733509(82)$ \\
\hline \multicolumn{10}{|c|}{${ }^{14} \mathrm{~N}$ nuclear quadrupole coupling constants ${ }^{\mathrm{b}} / \mathrm{MHz}$} \\
\hline $1.5\left(\chi_{\mathrm{aa}}\right)$ & $-6.2977(26)$ & $-6.286(13)$ & $-6.290(10)$ & $-6.300(14)$ & $-6.306(16)$ & $-6.284(13)$ & $-6.297(22)$ & $-6.293(20)$ & \\
\hline $\begin{array}{l}0.25\left(\chi_{\mathrm{bb}}-\right. \\
\left.\chi_{\mathrm{cc}}\right)\end{array}$ & $0.09447(76)$ & $0.0983(34)$ & $0.0984(26)$ & $0.1014(38)$ & $0.0945(42)$ & $0.0981(33)$ & $0.1008(35)$ & $0.0963(36)$ & \\
\hline \multicolumn{10}{|c|}{ Centrifugal distortion constants ${ }^{\mathrm{c}} / \mathrm{kHz}$} \\
\hline$\Delta_{\mathrm{J}}$ & $0.013523(63)$ & $0.0147(12)$ & $0.0142(10)$ & $0.0131(14)$ & $0.0124(15)$ & $0.0137(12)$ & $0.0142(13)$ & $0.0143(13)$ & $0.01218(43)$ \\
\hline$\Delta_{\mathrm{IK}}$ & $0.15751(50)$ & $0.162(15)$ & $0.150(11)$ & $0.135(16)$ & $0.129(18)$ & $0.138(14)$ & $0.140(18)$ & $0.144(17)$ & $0.1509(80)$ \\
\hline$\Delta_{\mathrm{K}}$ & $0.719(63)$ & 0.719 & 0.719 & 0.719 & 0.719 & 0.719 & 0.719 & 0.719 & 0.719 \\
\hline$\delta_{j}$ & $0.003416(39)$ & $0.00409(61)$ & $0.00375(50)$ & $0.00343(66)$ & $0.00275(74)$ & $0.00359(59)$ & $0.00377(64)$ & $0.00394(62)$ & $0.00285(23)$ \\
\hline$\delta_{\mathrm{k}}$ & $0.1066(24)$ & $0.138(38)$ & $0.119(31)$ & $0.099(41)$ & $0.076(47)$ & $0.088(37)$ & $0.114(42)$ & $0.109(41)$ & $0.081(14)$ \\
\hline $\mathrm{rms} / \mathrm{kHz}$ & 2.3 & 1.7 & 1.3 & 1.8 & 2.0 & 1.6 & 1.7 & 1.7 & 0.5 \\
\hline
\end{tabular}

${ }^{a}$ Calculated rotational constants $(\mathrm{MP} 2 / 6-311++\mathrm{G}(2 \mathrm{~d}, 2 \mathrm{p}))$ from this work: $\mathrm{A}=2926.211 \mathrm{MHz}, \mathrm{B}=928.314 \mathrm{MHz}, \mathrm{C}=704.741 \mathrm{MHz}$

${ }^{\mathrm{b}}$ Calculated ${ }^{14} \mathrm{~N}$ hyperfine constants from this work: B3LYP/6-311++G(2d,2p): $1.5\left(\chi_{\mathrm{aa}}\right)=-6.331 \mathrm{MHz}, 0.25\left(\chi_{\mathrm{bb}}-\chi_{\mathrm{cc}}\right)=0.120 \mathrm{MHz}$, $\chi_{\mathrm{ab}}=0.764 \mathrm{MHz} ; \mathrm{MP} 2 / 6-311++\mathrm{G}(2 \mathrm{~d}, 2 \mathrm{p}): 1.5\left(\chi_{\mathrm{aa}}\right)=-5.773 \mathrm{MHz}, \chi_{\mathrm{ab}}=-0.661 \mathrm{MHz}$.

${ }^{c}$ Some centrifugal distortion constants for minor isotopologues were held fixed to the parent values during the fit. These are given here without uncertainties. 
Table 7: Equilibrium $\left(\mathrm{r}_{\mathrm{e}}\right)(\mathrm{MP} 2 / 6-311 \mathrm{G}++(2 \mathrm{~d}, 2 \mathrm{p}))$, substitution $\left(\mathrm{r}_{\mathrm{s}}\right)^{\mathrm{a}}$ and ground state effective $\left(\mathrm{r}_{\mathrm{o}}\right)$ structural parameters of BN, 2FBN, $3 F B N, 23 D F B N, 24 D F B N$ and PFBN) determined in this work. Bond lengths are in $\AA$ and angles in degrees.

\begin{tabular}{|c|c|c|c|c|c|c|c|c|c|}
\hline & \multicolumn{3}{|c|}{ benzonitrile ${ }^{\mathrm{b}}$} & \multicolumn{3}{|c|}{ 2-fluorobenzonitrile } & \multicolumn{3}{|c|}{ 3-fluorobenzonitrile } \\
\hline & $\mathrm{r}_{\mathrm{e}}$ & $\mathrm{r}_{\mathrm{s}}$ & $r_{o}$ & $r_{e}$ & $\mathrm{r}_{\mathrm{s}}$ & $r_{o}$ & $\mathrm{r}_{\mathrm{e}}$ & $\mathrm{r}_{\mathrm{S}}$ & $r_{o}$ \\
\hline $\mathrm{C}_{1}-\mathrm{C}_{2}$ & 1.401 & $1.381(6)$ & $1.397(7)$ & 1.396 & $1.357(7)$ & $1.383(4)$ & 1.401 & $1.393(14)$ & $1.398(10)$ \\
\hline $\mathrm{C}_{2}-\mathrm{C}_{3}$ & 1.393 & $1.417(12)$ & $1.397(8)$ & 1.386 & $1.423(14)$ & $1.384(2)$ & 1.386 & $1.386(14)$ & $1.388(7)$ \\
\hline $\mathrm{C}_{3}-\mathrm{C}_{4}$ & 1.396 & $1.396(1)$ & $1.398(6)$ & 1.394 & $1.377(20)$ & $1.397(4)$ & 1.388 & $1.380(4)$ & $1.386(3)$ \\
\hline $\mathrm{C}_{4}-\mathrm{C}_{5}$ & & & & 1.397 & $1.417(23)$ & $1.397(4)$ & 1.395 & $1.398(2)$ & $1.396(4)$ \\
\hline $\mathrm{C}_{5}-\mathrm{C}_{6}$ & & & & 1.391 & $1.399(4)$ & $1.394(3)$ & 1.393 & $1.389(3)$ & $1.397(6)$ \\
\hline $\mathrm{C}_{6}-\mathrm{C}_{1}$ & & & & 1.403 & $1.396(4)$ & $1.402(5)$ & 1.401 & $1.393(13)$ & $1.392(11)$ \\
\hline $\mathrm{C}_{7}-\mathrm{C}_{1}$ & 1.435 & $1.450(3)$ & $1.445(8)$ & 1.431 & $1.449(4)$ & $1.443(5)$ & 1.435 & $1.447(5)$ & $1.447(5)$ \\
\hline $\mathrm{N}-\mathrm{C}_{7}$ & 1.173 & $1.158(1)$ & $1.158(3)$ & 1.173 & $1.158(1)$ & $1.157(1)$ & 1.173 & $1.158(2)$ & $1.158(3)$ \\
\hline$\angle\left(\mathrm{C}_{1}-\mathrm{C}_{2}-\mathrm{C}_{3}\right)$ & 119.5 & $118.1(5)$ & $119.1(5)$ & 121.8 & $120.2(7)$ & $122.1(2)$ & 118.0 & $117.0(5)$ & $117.1(5)$ \\
\hline$\angle\left(\mathrm{C}_{2}-\mathrm{C}_{3}-\mathrm{C}_{4}\right)$ & 120.3 & $120.2(1)$ & $120.1(3)$ & 118.9 & $119.2(6)$ & $118.4(2)$ & 122.7 & $123.3(3)$ & $123.2(2)$ \\
\hline$\angle\left(\mathrm{C}_{3}-\mathrm{C}_{4}-\mathrm{C}_{5}\right)$ & 120.0 & $120.1(1)$ & $120.2(6)$ & 120.4 & $120.3(2)$ & $120.6(1)$ & 118.5 & $118.2(1)$ & $118.3(2)$ \\
\hline$\angle\left(\mathrm{C}_{4}-\mathrm{C}_{5}-\mathrm{C}_{6}\right)$ & & & & 120.1 & $119.7(4)$ & $120.0(1)$ & 120.7 & $120.5(1)$ & $120.6(2)$ \\
\hline$\angle\left(\mathrm{C}_{5}-\mathrm{C}_{6}-\mathrm{C}_{1}\right)$ & & & & 120.1 & $119.0(2)$ & $119.6(3)$ & 119.3 & $119.2(2)$ & $119.0(9)$ \\
\hline$\angle\left(\mathrm{C}_{6}-\mathrm{C}_{1}-\mathrm{C}_{2}\right)$ & 120.5 & $123.4(9)$ & $121.4(5)$ & 118.7 & $121.6(6)$ & $119.2(2)$ & 120.8 & $121.7(5)$ & $121.8(8)$ \\
\hline$\angle\left(\mathrm{C}_{7}-\mathrm{C}_{1}-\mathrm{C}_{2}\right)$ & 119.8 & 118.3(1) & $119.3(7)$ & 120.4 & $119.0(6)$ & $120.7(3)$ & 119.4 & $118.8(8)$ & $118.6(9)$ \\
\hline$\angle\left(\mathrm{N}-\mathrm{C}_{7}-\mathrm{C}_{1}\right)$ & 180 & 180 & 180 & 178.8 & $179.1(3)$ & $179.2(3)$ & 179.8 & $179.8(6)$ & $179.7(1.0)$ \\
\hline $\mathrm{D}\left(\mathrm{N}-\mathrm{C}_{7}-\mathrm{C}_{1}-\mathrm{C}_{2}\right)$ & 0 & 0 & 0 & 180 & 180 & 180 & 180 & 180 & 180 \\
\hline \multirow[t]{3}{*}{$\sigma\left(\times 10^{3}\right)$} & & & 9.3 & & & 3.6 & & & 6.3 \\
\hline & \multicolumn{3}{|c|}{ 2,3-difluorobenzonitrile } & \multicolumn{3}{|c|}{ 2,4-difluorobenzonitrile } & \multicolumn{3}{|c|}{ pentafluorobenzonitrile } \\
\hline & $\mathrm{r}_{\mathrm{e}}$ & $\mathrm{r}_{\mathrm{s}}$ & $\mathrm{r}_{\mathrm{o}}$ & $\mathrm{r}_{\mathrm{e}}$ & $\mathrm{r}_{\mathrm{S}}$ & $\mathrm{r}_{\mathrm{o}}$ & $\mathrm{r}_{\mathrm{e}}$ & $\mathrm{r}_{\mathrm{S}}$ & $\mathrm{r}_{\mathrm{o}}$ \\
\hline $\mathrm{C}_{1}-\mathrm{C}_{2}$ & 1.395 & $1.375(11)$ & $1.386(7)$ & 1.396 & $1.387(4)$ & $1.385(6)$ & 1.397 & $1.400(2)$ & $1.400(2)$ \\
\hline $\mathrm{C}_{2}-\mathrm{C}_{3}$ & 1.391 & $1.391(14)$ & $1.389(3)$ & 1.385 & $1.357(5)$ & $1.376(5)$ & 1.388 & $1.359(3)$ & $1.372(3)$ \\
\hline $\mathrm{C}_{3}-\mathrm{C}_{4}$ & 1.386 & $1.366(8)$ & $1.381(4)$ & 1.387 & $1.370(13)$ & $1.393(4)$ & 1.392 & $1.391(1)$ & $1.393(2)$ \\
\hline $\mathrm{C}_{4}-\mathrm{C}_{5}$ & 1.396 & $1.400(2)$ & $1.398(4)$ & 1.389 & $1.409(15)$ & $1.386(4)$ & & & \\
\hline $\mathrm{C}_{5}-\mathrm{C}_{6}$ & 1.391 & $1.387(3)$ & $1.396(6)$ & 1.390 & $1.393(25)$ & $1.398(16)$ & & & \\
\hline $\mathrm{C}_{6}-\mathrm{C}_{1}$ & 1.403 & $1.409(4)$ & $1.404(8)$ & 1.402 & $1.403(15)$ & $1.402(11)$ & & & \\
\hline
\end{tabular}




\begin{tabular}{|c|c|c|c|c|c|c|c|c|c|}
\hline $\mathrm{C}_{7}-\mathrm{C}_{1}$ & 1.431 & $1.438(2)$ & $1.437(4)$ & 1.430 & $1.440(1)$ & $1.437(4)$ & 1.426 & $1.435(1)$ & $1.434(3)$ \\
\hline $\mathrm{N}-\mathrm{C}_{7}$ & 1.173 & $1.158^{\mathrm{c}}$ & $1.158^{\mathrm{d}}$ & 1.173 & $1.158(1)$ & $1.158(2)$ & 1.173 & $1.157(1)$ & $1.156(2)$ \\
\hline$\angle\left(\mathrm{C}_{1}-\mathrm{C}_{2}-\mathrm{C}_{3}\right)$ & 120.0 & $119.6(5)$ & $119.9(3)$ & 122.1 & $123.5(3)$ & $122.9(3)$ & 120.9 & $121.6(2)$ & $121.3(5)$ \\
\hline$\angle\left(\mathrm{C}_{2}-\mathrm{C}_{3}-\mathrm{C}_{4}\right)$ & 120.9 & $121.8(5)$ & $121.3(3)$ & 117.5 & $117.4(4)$ & $116.8(3)$ & 119.6 & $119.8(1)$ & $119.7(2)$ \\
\hline$\angle\left(\mathrm{C}_{3}-\mathrm{C}_{4}-\mathrm{C}_{5}\right)$ & 119.2 & $118.9(1)$ & $119.0(2)$ & 122.7 & $122.8(2)$ & $123.2(7)$ & 120.4 & $119.8(1)$ & $120.1(2)$ \\
\hline$\angle\left(\mathrm{C}_{4}-\mathrm{C}_{5}-\mathrm{C}_{6}\right)$ & 120.5 & $120.3(1)$ & $120.4(2)$ & 118.5 & $117.9(3)$ & $118.2(1)$ & & & \\
\hline$\angle\left(\mathrm{C}_{5}-\mathrm{C}_{6}-\mathrm{C}_{1}\right)$ & 119.9 & $119.7(2)$ & $119.5(5)$ & 120.7 & $120.0(7)$ & $120.1(8)$ & & & \\
\hline$\angle\left(\mathrm{C}_{6}-\mathrm{C}_{1}-\mathrm{C}_{2}\right)$ & 119.5 & $119.7(5)$ & $119.8(8)$ & 118.5 & $118.4(9)$ & $118.8(4)$ & 118.6 & $117.4(2)$ & $118.0(4)$ \\
\hline$\angle\left(\mathrm{C}_{7}-\mathrm{C}_{1}-\mathrm{C}_{2}\right)$ & 119.6 & $121.0(5)$ & $120.1(6)$ & 120.5 & $121.7(8)$ & $121.2(6)$ & 120.7 & $121.3(2)$ & $121.0(3)$ \\
\hline$\angle\left(\mathrm{N}-\mathrm{C}_{7}-\mathrm{C}_{1}\right)$ & 178.8 & $179.1(3)$ & $179.5(7)$ & 178.9 & $179.2(3)$ & $179.3(5)$ & 180 & 180 & 180 \\
\hline $\mathrm{D}\left(\mathrm{N}-\mathrm{C}_{7}-\mathrm{C}_{1}-\mathrm{C}_{2}\right)$ & 180 & 180 & 180 & 180 & 180 & 180 & 0 & 0 & 0 \\
\hline$\sigma\left(\times 10^{3}\right)$ & & & 6.3 & & & 5.1 & & & 7.0 \\
\hline \multicolumn{10}{|c|}{$\begin{array}{l}\text { a Note that while the Kraitchman equations provide only absolute values of the coordinates, the signs are inferred based on the orientation of the } \\
\text { conformer in its principal axis system based on the ab initio predictions. } \\
\text { b Rotational constants from ref. } 16 \text { used to derived the } \mathrm{r}_{\mathrm{s}} \text { and } \mathrm{r}_{\mathrm{o}} \text { structures including deuterium isotopologues. } \\
{ }^{\mathrm{c}} \text { Due to large Costain error, the } \mathrm{r}_{\mathrm{o}} \text { parameter was used. } \\
\text { d } \mathrm{C}-\mathrm{N} \text { bond length was fixed to the average values of the other benzonitriles. }\end{array}$} \\
\hline
\end{tabular}


Table 8: Results from the ${ }^{14} \mathrm{~N}$ hyperfine analysis including the angle $\theta$ between the least principal inertial axis and the $\mathrm{C} \equiv \mathrm{N}$ bond, nuclear quadrupole coupling constants in the principal coupling tensor frame, and the ionic character $\left(\pi_{\mathrm{c}}, \pi_{\mathrm{x}}\right.$ and $\left.\pi_{\mathrm{y}}\right)$ at nitrogen in BN and related molecules. ${ }^{\mathrm{a}, \mathrm{b}}$

\begin{tabular}{|c|c|c|c|c|c|c|c|c|}
\hline Species & $\theta\left(^{\circ}\right)$ & $\chi_{\mathrm{zz}}(\mathrm{MHz})$ & $\chi_{\mathrm{xx}}(\mathrm{MHz})$ & $\chi_{\text {yy }}(\mathrm{MHz})$ & $\chi_{\mathrm{xx}}-\chi_{\mathrm{yy}}$ & $\pi_{\mathrm{c}}$ & $\pi_{\mathrm{x}}$ & $\pi_{\mathrm{y}}$ \\
\hline benzonitrile & 0 & -4.237 & 2.288 & 1.949 & 0.339 & 0.270 & 0.122 & 0.148 \\
\hline 2-fluorobenzonitrile & 7.1 & -4.281 & 2.311 & 1.970 & 0.341 & 0.262 & 0.118 & 0.144 \\
\hline 3-fluorobenzonitrile & $\begin{array}{c}17.3 \\
(17.3)\end{array}$ & $\begin{array}{l}-4.257 \\
(-4.27)\end{array}$ & $\begin{array}{l}2.272 \\
(2.28)\end{array}$ & 1.990 & 0.282 & $\begin{array}{c}0.267 \\
(0.264)\end{array}$ & $\begin{array}{c}0.123 \\
(0.121)\end{array}$ & \begin{tabular}{|c|}
0.144 \\
$(0.143)$
\end{tabular} \\
\hline 2,3-difluorobenzonitrile & 15.3 & -4.322 & 2.324 & 1.998 & 0.326 & 0.255 & 0.115 & 0.140 \\
\hline 2,4-difluorobenzonitrile & 6.4 & -4.279 & 2.369 & 1.910 & 0.459 & 0.263 & 0.114 & 0.149 \\
\hline pentafluorobenzonitrile & 0 & -4.387 & 2.410 & 1.977 & 0.433 & 0.243 & 0.105 & 0.138 \\
\hline
\end{tabular}




\section{Figure Captions}

Figure 1: Geometries of the benzonitriles in their principal axis systems based on the ab initio (MP2/6-311++G(2d,2p) structures.

Figure 2: A portion of the cp-FTMW spectrum (60 000 cycles) of PFBN showing the $10_{010}-9_{09}$ and $10_{110-9}{ }_{19}$ rotational transitions for the parent and some minor isotopologues in natural abundance.

Figure 3: FTMW spectrum (100 cycles) of the $10_{010}-9_{09}$ and $10_{110}-9_{19}$ rotational transition of PFBN showing the hyperfine splitting arising from the ${ }^{14} \mathrm{~N}$ quadrupolar nucleus.

Figure 4: Possible resonance structures for $2 \mathrm{FBN}$ and $3 \mathrm{FBN}$ involving donation of the lone pair on fluorine to the $\pi$ orbitals of the aromatic ring. Note that two additional resonance contributors are possible for $2 \mathrm{FBN}$ that are similar to the center drawing but with the negative charge on $\mathrm{C} 3$ or C5. 
Figure 1: Kamaee, Sun, Luong and van Wijngaarden
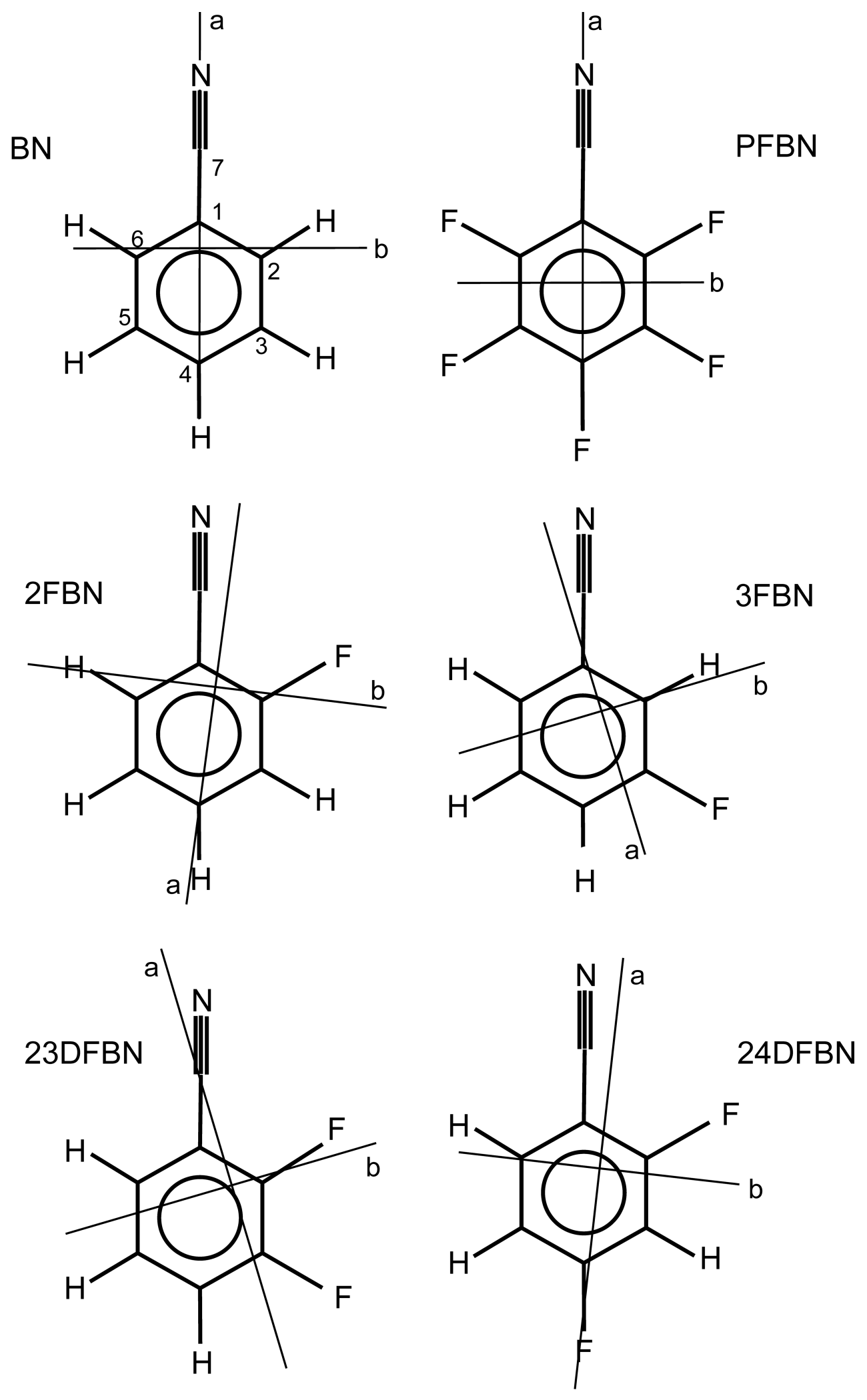
Figure 2: Kamaee, Sun, Luong and van Wijngaarden

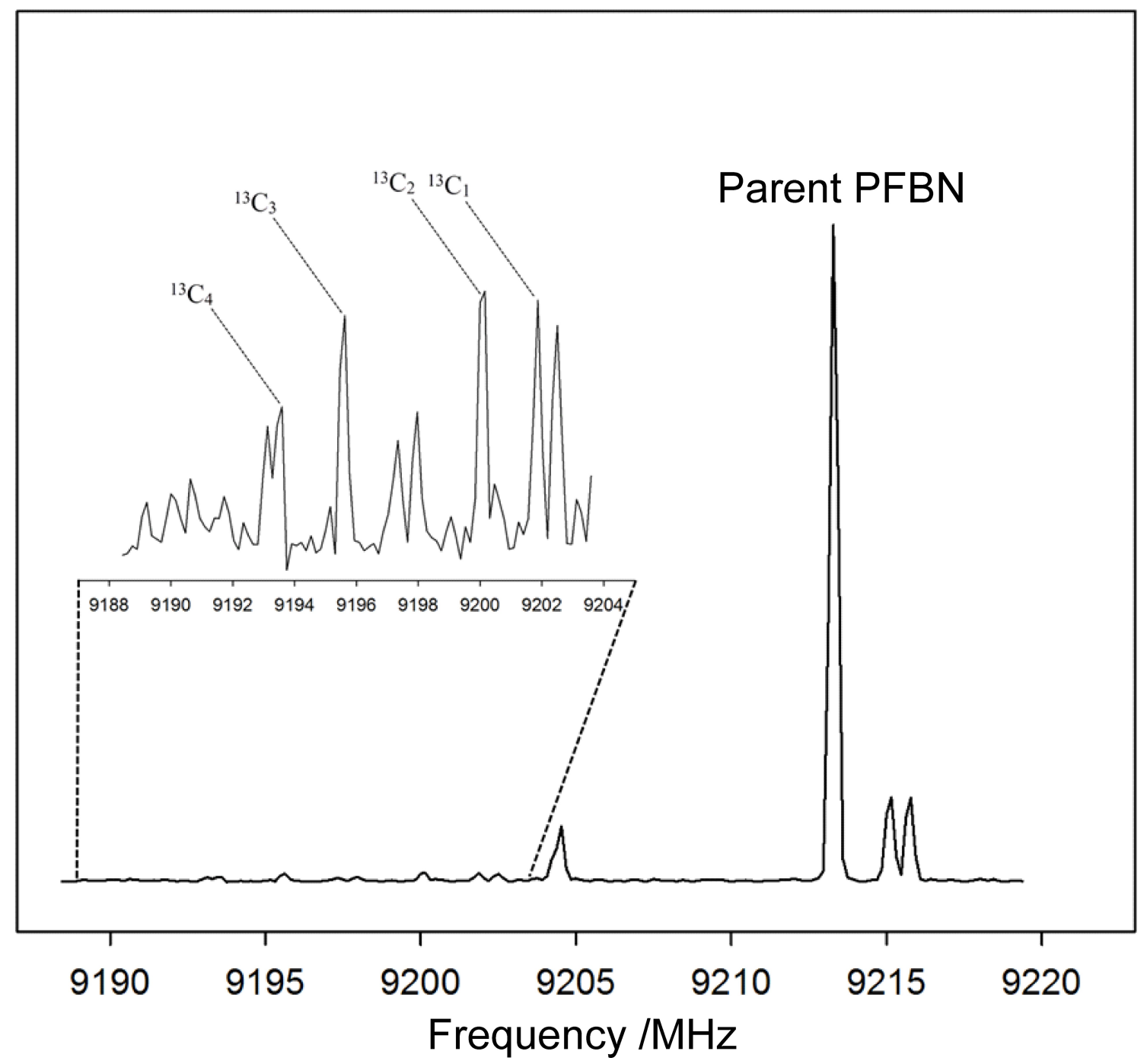


Figure 3: Kamaee, Sun, Luong and van Wijngaarden

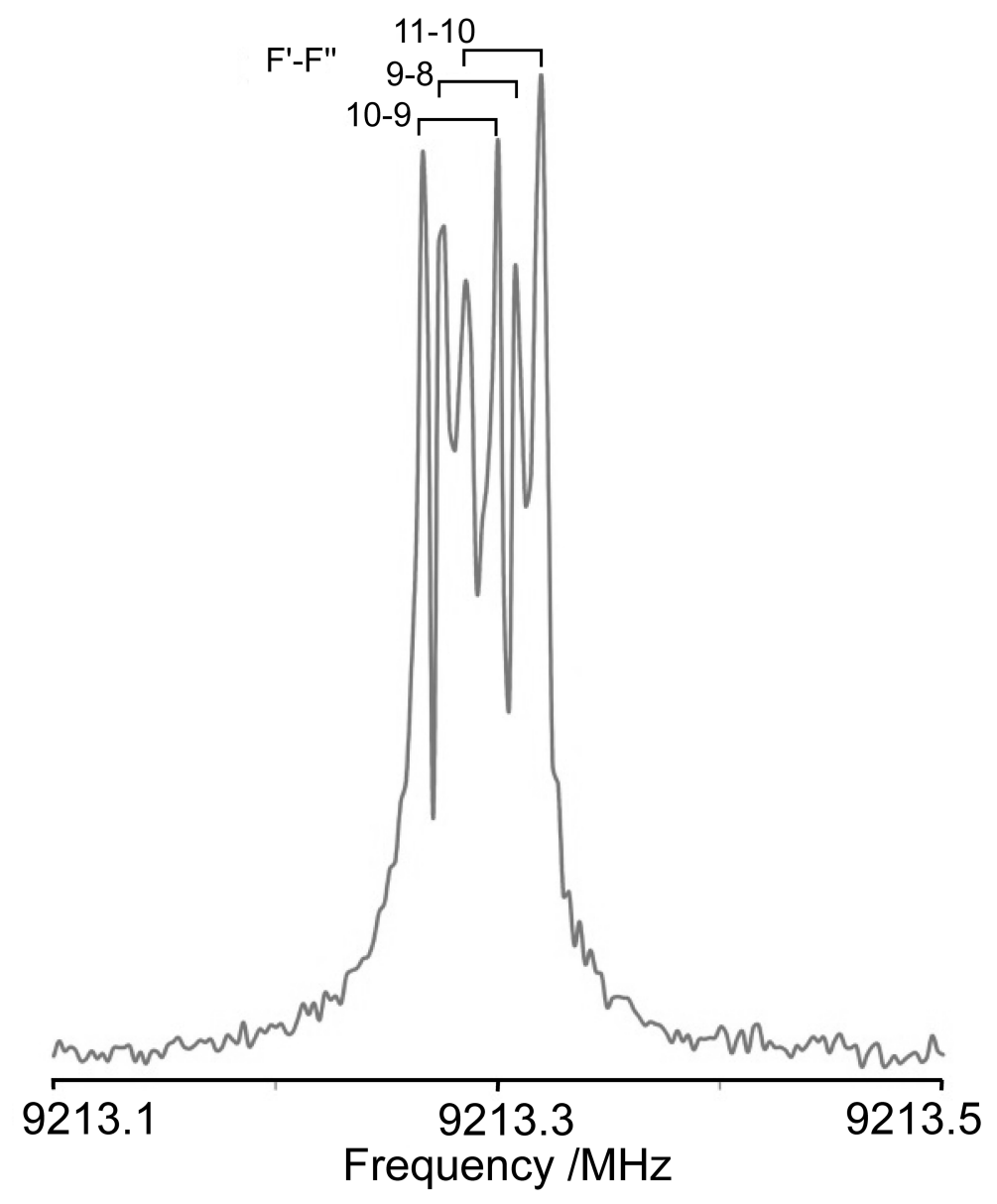


Figure 4: Kamaee, Sun, Luong and van Wijngaarden

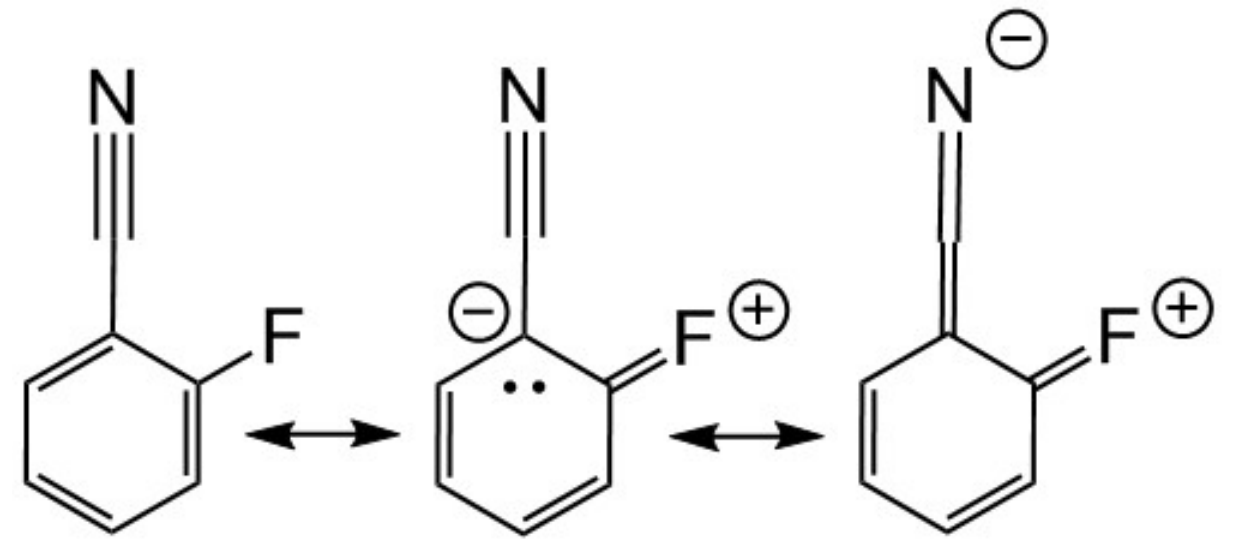

\section{FBN}

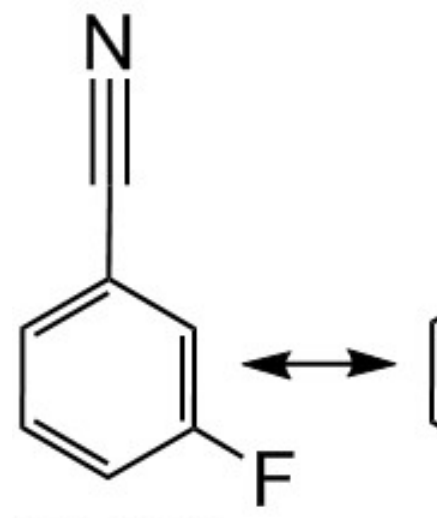

3FBN

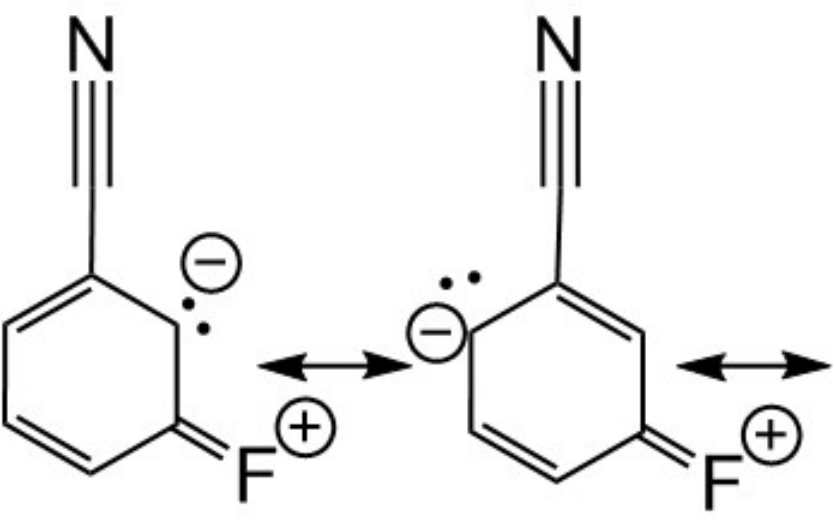

(†)

.

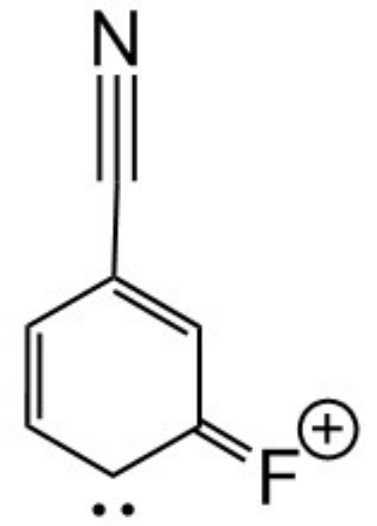

$\Theta$ 


\section{References}

${ }^{1}$ O'Hagan, D. Understanding Organofluorine Chemistry. An Introduction to the C-F Bond. Chem. Soc. Rev. 2008, 37, 308-319.

${ }^{2}$ Berger, R.; Resnati, G.; Metrangolo, P.; Weber, E.; Hulliger, J. Organic Fluorine Compounds:

A Great Opportunity for Enhanced Materials Properties. Chem. Soc. Rev. 2011, 40, 3496-3508.

${ }^{3}$ Müller, K.; Faeh, C.; Diederich, F. Fluorine in Pharmaceuticals: Looking Beyond Intuition.

Science. 2007, 317, 1881-1886.

${ }^{4}$ Jeschke, P. The Unique Role of Fluorine in the Design of Active Ingredients for Modern Crop

Protection. Chem. Bio. Chem. 2004, 5, 570-589.

${ }^{5}$ Kisiel, Z.; Białkowska-Jaworska, E.; Pszczółkowski, L. The Millimeter-Wave Rotational

Spectrum of Fluorobenzene. J. Mol. Spectrosc. 2005, 232, 47-54.

${ }^{6}$ Nygaard, L.; Bojesen, I.; Pedersen, T.; Rastrup-Andersen, J. Structure of Fluorobenzene. J. Mol.

Struct. 1968, 2, 209-215.

${ }^{7}$ Doraiswamy, S.; Sharma, S. D. $\mathrm{R}_{\mathrm{o}}$ Geometries of Fluorobenzenes and Distortions in Benzene Ring Structure on Substitution. J. Mol. Struct. 1983, 102, 81-92.

${ }^{8}$ van Dijk, C.W.; Sun, M.; van Wijngaarden, J. Microwave Rotational Spectra and Structures of 2-Fluorpyridine and 3-Fluoropyridine. J. Phys. Chem. A. 2012, 116, 4082-4088.

${ }^{9}$ van Dijk, C.W.; Sun, M.; van Wijngaarden, J. Investigation of Structural Trends in Difluoropyridine Rings Using Chirped-Pulse Fourier Transform Microwave Spectroscopy and Ab Initio Calculations. J. Mol. Spectrosc. 2012, 280, 34-41.

${ }^{10}$ Wohlfart, K.; Schnell, M.; Grabow J.-U.; Küpper, J. Precise Dipole Moment and Quadrupole Coupling Constants of Benzonitrile, J. Mol. Spectrosc. 2008, 247, 119-121. 
${ }^{11}$ Campanelli, A.R.; Domenicano, A.; Ramondo F.; I. Hargittai, I. Molecular Structure and Benzene Ring Deformation of Three Cyanobenzenes from Gas Phase Electron Diffraction and Quantum Chemical Calculations. J. Phys. Chem. A. 2008, 112, 10998-11008.

${ }^{12}$ Rudolph, H.D.; Demaison, J.; Császár, A.G. Accurate Determination of the Deformation of the Benzene Ring Upon Substitution: Equilibrium Structures of Benzonitrile and Phenlyacetylene. $J$. Phys. Chem. A. 2013, 117, 12969-12982.

${ }^{13}$ Lide, D.R. Microwave Spectrum and Structure of Benzonitrile. J. Chem. Phys. 1954, 22, $1577-$ 1578.

${ }^{14}$ Erlandsson, G. Microwave Spectrum of Benzonitrile. J. Chem. Phys. 1954, 22, 1152-1152.

${ }^{15}$ Wlodarczak, G.; Burie, J.; Demaison, J.; Vormann, K.; Császár, A.G. The Rotatational Spectrum of Benzonitrile: Experimental and Theoretical Determination of the Quartic Centrifugal Distortion Constants. J. Mol. Spectrosc. 1989, 134, 297-304.

${ }^{16}$ Bak, B.; Christensen, D.; Dixon, W.B.; Hansen-Nygaard, L.; Rastrup-Andersen, J. Benzene Ring Distortion By One Substituent- Microwave Determination of Complete Structure of Benzonitrile, J. Chem. Phys. 1962, 37, 2027-2031.

${ }^{17}$ Casado, J.; Nygaard, L.; Sørensen, G.O. Microwave Spectra of Isotopic Benzonitriles- Refined Molecular Structure of Benzonitrile. J. Mol. Struct. 1971, 8, 211-224.

${ }^{18}$ Fliege, E.; Betmann, G.; Schwarz, R.; Dreizler, H. Quadrupole Coupling in Benzonitrile-An Application of Microwave Fourier Transform Spectroscopy. Z. Naturforsch. A. 1981, 36, 11241125.

${ }^{19}$ Vormann, K.; Andresen, U.; Heineking, N.; Dreizler, H. Quadrupole Hyperfine Structure in the Rotational Spectrum of Benzonitrile. Z. Naturforsch. 1988, 47a, 283-284. 
${ }^{20}$ Dahmen, U.; Stahl, W.; Dreizler, H. The Rotational Spectrum of the Benzonitrile-Ar Van der Waals Complex, Ber. Bunsenges. Phys. Chem. 1994, 98, 970-974.

${ }^{21}$ Schmitz, D.; Shubert, V.A.; Betz, T.; Schnell, M. Multi-Resonance Effects Within a Single Chirp in Broadband Rotational Spectroscopy: The Rapid Passage Regime for Benzonitrile. $J$. Mol. Spectrosc. 2012, 280, 77-84.

${ }^{22}$ Wohlfart, K.; Grätz, F.; Filsinger, F.; Haak, H.; Meijer, G.; Küpper, J, Alternate-Gradient Focusing and Deceleration of Large Molecules. Phys. Rev. A: At., Mol., Opt. Phys. 2008, 77, 031404

${ }^{23}$ Patterson, D.; Doyle, J.M. A Slow, Continuous Beam of Cold Benzonitrile. Phys. Chem. Chem. Phys. B, 2015, 17, 5372-5375.

${ }^{24}$ Sharma, S.D.; Doraiswamy, S. Microwave Spectrum of Pentafluorobenzonitrile. P. Indian AS. 1968, $67,12-18$.

${ }^{25}$ Krüger, M.; Dreizler, H. The Microwave Spectra and Nitrogen Quadrupole Coupling Constants of Cyano- and Isocyano-Pentafluorobenzene. Z. Naturforsch. 1992, 47a, 865-868.

${ }^{26}$ Böttcher, O.; Sutter, D.H. Rotational Spectrum and ${ }^{14} \mathrm{~N}-$ Quadrupole Coupling Constants of Orthofluorobenzonitrile, A Microwave Fourier Transform Study. Z. Naturforsch. 1986, 41a, 955-958.

${ }^{27}$ Dutta, A.; Jaman, A.I.; Nandi, R.N. Microwave Spectral Study of 2-Fluorobenzonitrile. J. Mol. Spectrosc. 1987, 124, 486-488.

${ }^{28}$ Böttcher, O.; Sutter, D.H. Nitrogen Quadrupole Hyperfine Structure in the Rotational Spectrum of 2-, 3-, and 4-Fluoro-Benzonitrile. A Comparative High Resolution Microwave Fourier Transform Study. Z. Naturforsch. 1987, 43a, 47-58. 
${ }^{29}$ Dutta, A.; Jaman, A.I.; Ghosh, D.K.; Nandi R.N. Microwave Spectral Study of 3Fluorobenzonitrile. J. Mol. Spectrosc. 1986, 118, 232-236.

${ }^{30}$ Bak, B.; Christensen, D.H.; Kristiansen, N.A.; Nicolaisen, F.; Nielson, O.F. Preliminary Structure and Force Field of 4-Fluoro-Benzonitrile from Microwave, Raman and Infrared Spectra. Acta Chem. Scand. A. 1983, 37, 601-607.

${ }^{31}$ Böttcher, O.; Sutter, D.H. Quadrupole Coupling in 4-Fluoro-Benzonitrile. A Microwave Fourier Transform Study. Z. Naturforsch. 1986, 41a, 753-755.

${ }^{32}$ Varadwaj, P.R.; Jaman, A.I. Centrifugal Distortion Analysis of the Millimeter-Wave Spectrum of 2-Fluorobenzonitrile and Ab Initio DFT Calculations. J. Mol. Spectrosc. 2006, 236, 70-74.

${ }^{33}$ Maiti, S.; Jaman, A.I.; Dutta, A.; Nandi, R.N. Microwave Spectrum of 2,3-

Difluorobenzonitrile. J. Mol. Spectrosc. 1990, 140, 416-418.

${ }^{34}$ Varadwaj, P.R.; Jaman, A.I. Millimeter-Wave Spectrum of 2,3-Difluorobenzonitrile and Ab Initio DFT Calculations. J. Mol. Spectrosc. 2006, 239, 216-220.

${ }^{35}$ Sharma, S.D.; Doraiswamy, S. Microwave Spectrum of 2,6-Difluorobenzonitrile. J. Mol. Spectrosc. 1996, 180, 7-14.

${ }^{36}$ Onda, M.; Kasagi, T.; Jaman A.I. Microwave Spectrum and Quadrupole Coupling Constants of 2,3-Difluorobenzonitrile. J. Mol. Struct. 2002, 612, 167-170.

${ }^{37}$ Sedo, G.; van Wijngaarden, J. J. Chem. Phys. 2009, 131, 044303.

${ }^{38}$ Evangelisti, L.; Sedo, G.; van Wijngaarden, J. Rotational Spectrum of 1,1,1,-Trifluoro-2butanone Using Chirped-Pulse Fourier Transform Microwave Spectroscopy. J. Phys. Chem. A. 2011, $115,685-690$.

${ }^{39}$ Pickett, H.M. The Fitting and Prediction of Vibration-Rotational Spectra With Spin Interactions. J. Mol. Spectrosc. 1991, 148, 371-377. 
${ }^{40}$ Gaussian 09, Revision B.01, Frisch, M. J. et al. Gaussian, Inc., Wallingford CT, 2009.

${ }^{41}$ NBO 5.0. Glendening, E.D.; Badenhoop, J.K.; Reed, A.E.; Carpenter, J.E.; Bohmann, J.A.;

Morales, C.M.; Weinhold, F. (Theoretical Chemistry Institute, University of Wisconsin, Madison, WI, 2001); http://www.chem.wisc.edu/ nbo5

${ }^{42}$ Kraitchman, J. Determination of Molecular Structure from Microwave Spectroscopic Data. Am. J. Phys. 1953, 21, 17-24.

${ }^{43}$ Kisiel, Z. PROSPE - Programs for Rotational SPEctroscopy, Sept 1, 2015. $<$ http://info.ifpan.edu.pl/ $\sim$ kisiel/prospe.htm $>$.

${ }^{44}$ Costain, C.C. Further Comments on the Accuracy of $r_{s}$ Substitution Structures. Trans. Am. Crystallogr. Assoc. 1966, 2, 157.

${ }^{45}$ Townes, C.H.; Schawlow, A.L. Microwave Spectroscopy. Dover, New York, 1975.

${ }^{46}$ Gordy W.; Cook, R.L. Microwave Molecular Spectra; Wiley: New York, 1984 and references therein.

${ }^{47}$ Bent, H.A. An Appraisal of Valence-Bon Structures and Hybridization in Compounds of the First-Row Elements. Chem. Rev. 1961, 61, 275-311.

${ }^{48}$ Olsen, J.; Seiler, P.; Wagner, B.; Fischer, H.; Tschopp, T.; Obst-Sander, U.; Banner, D.W.; Kansy, M.; Muller, K.; Diederich, F. A Fluorine Scan of the the Phenylamidinium Needle of Tricyclic Thrombin Inhibitors: Effects of Fluorine Substitution on pKa and Binding Affinity and Evidence of Intermolecular C-F..CN Interactions. Org. Biomol. Chem. 2004, 2, 1339-1352. 
TOC graphic

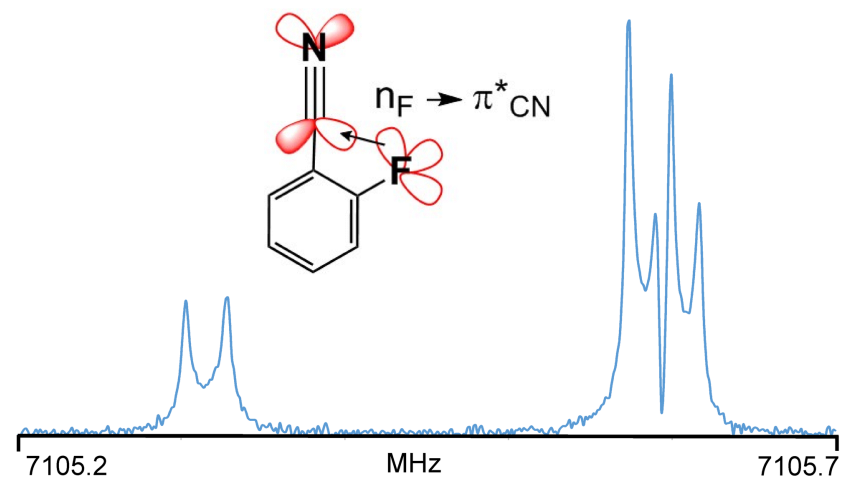

